\title{
The Effect of Temperature on the Braking Force Experienced by Magnet Falling Through a Copper Pipe
}

\author{
Miyu Bansal ${ }^{1}$ and John Duivestein ${ }^{1}$ \\ ${ }^{1}$ Glenunga International High School, Glenunga, Australia
}

\begin{abstract}
$\underline{\text { ABSTRACT }}$
The braking experienced by a magnet falling through a conductive pipe is often shown in laboratories, as it is highly intriguing and captures the imagination of students. The Eddy current is the physical phenomenon behind Eddy current braking, which has a lot of utility as Eddy current brakes do not utilise friction and hence do not wear. Linear eddy current brakes, often used on rail vehicles, use the rail as a conductor. Consequently, it is important to address the impact of the rail's properties. Previous papers have explored the significance of thickness and material however temperature has yet to be considered. Here, coils were wrung around a copper pipe and an oscilloscope was used to determine the position of a neodymium magnet falling through pipe at various temperatures in order to determine the magnet's terminal velocity. This data was then used to determine the braking force exerted by the pipe on the magnet. The experimental findings were compared to a theoretical model for the braking force. Graphing the inverse of the dragging constant and temperature showed a positive linear relationship suggesting that increasing the temperature reduces the braking force experienced by the magnet, which is in line with pre-existing theory that increasing the temperature will reduce conductivity, in turn reducing the eddy currents that cause the braking force. Finally, this study establishes that temperature, and hence the weather plays a significant role and needs to be considered when designing eddy current based machines, such as magnetic brakes in high-speed trains.
\end{abstract}

\section{Introduction}

Electricity and magnetism have always been an interest of mine, from a young age I was constructing basic circuits and playing with magnets, so it was natural for me to do an investigation into this area. Through my research, I came across many intriguing phenomena, one of which was eddy currents [1]. Eddy current brakes have many potential applications in high-speed electric trains, roller coasters. Their fast but smooth braking making them very unique and useful. It is important to note that the effectiveness of eddy current brakes is directly related to the speed of the moving component, with higher speeds leading to strong braking forces [2]. Interestingly, a large percentage of the power used in eddy current brakes is dissipated as heat. Consequently, circular eddy current brakes tend to have shorter lifespans than linear braking systems which utilise the rail instead of a circular disk. After reading about the application of eddy currents in induction brakes, which are used to smoothly stop high-speed trains, I began to wonder if the temperature of the rail track would affect the retarding force created by the eddy currents, specifically in linear eddy current brakes, see figure 1. Eddy current brakes apply Faraday's law of induction [4] and Lenz's law [5] by creating a significant retarding force that does not employ friction, instead using the electromagnetic forces between a magnet and a nearby conductor in relative motion. Dropping magnets down a conductive pipe is a simple and accurate method often used to explore eddy currents and since the magnet experiences a similar retarding force and follows the same physical laws as an eddy current brake, it is an ideal model to study the effect of temperature on eddy currents, see figure 2 . 


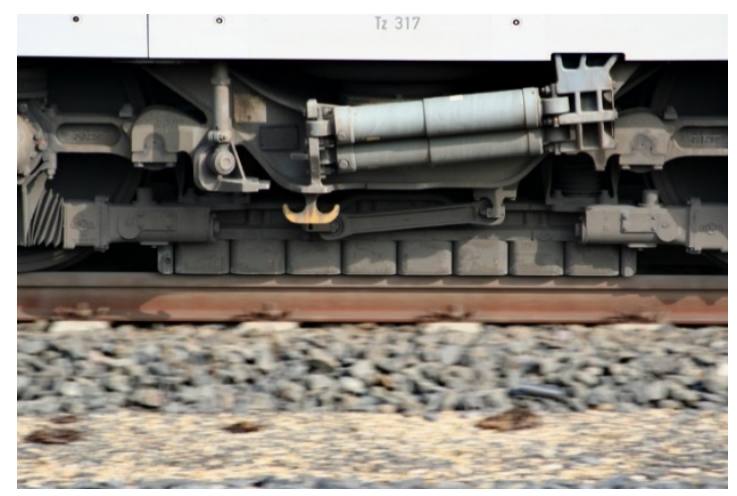

Figure 1. A linear eddy current brake in a German ICE 3 high-speed train in action [3]

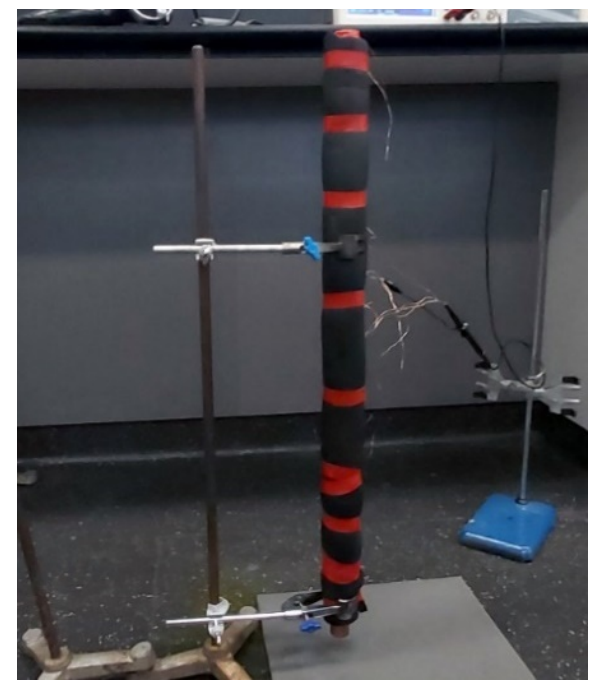

Figure 2. Experimental setup used to investigate effect of temperature on eddy currents

\section{Research Question:}

How does the retarding force experienced by a magnet falling down a copper tube change with the variation of the temperature of the copper pipe?

According to Faraday's law of induction [3], when a conductor experiences a change in magnetic flux, electromotive force $(\mathrm{emf}, \varepsilon)$ is induced within it, creating eddy currents. Since the magnitude of induced current is indirectly proportional to the resistance of the conductor [5], the resistance will have a significant impact on the eddy currents generated. Many research papers explore and mathematically model the impact of the thickness and material of the conductive pipe on the damping forces experienced by the magnet [7]-[11], but I was unable to find a paper that related the temperature dependence of the resistivity of copper and the retarding force experienced by a magnet falling through a copper pipe at different temperatures. Since, the resistance of copper linearly increases with its temperature over a range of $100-800 \mathrm{~K}[12,13]$ and the retarding force is directly proportional to the strength of the eddy currents, it seems reasonable that the retarding force will be greater at lower temperatures and lesser at higher temperatures.

The goal of my investigation was to prove or disprove my hypothesis through experimentation, and explain the results using physics principles. 


\section{Background Theory}

When a magnet falls down a copper pipe, its magnetic field moves with it and this moving magnetic field causes the pipe to experience a change in magnetic flux $\left(d \Phi_{B}\right)$. According to Faraday's law of induction [4], when the magnetic flux passing through a conductor changes, an emf is induced that is directly proportional to the rate of change of magnetic flux (Equation 1).

\section{Equation 1:}

$$
\varepsilon=-\frac{\mathrm{d} \Phi_{B}}{d t}
$$

The magnetic flux $\left(\Phi_{B}\right)$ through a surface is the perpendicular component of the magnetic field passing through the surface. According to [6] for a flat surface of area $\vec{A}$ with uniform magnetic field $\vec{B}$, the magnetic flux is given by Equation 2.

\section{Equation 2:}

$$
\Phi_{B}=\vec{B} \times \vec{A}=B \times A \times \phi ;
$$

where $\phi$ is the angle between magnetic field lines and normal to $A$, see figure 3 a.

a)

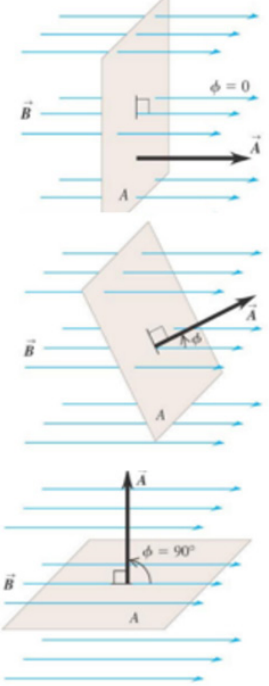

When the conductor is perpendicular to the magnetic field lines,$\phi=0$.

$\therefore \Phi_{B}=B \times A \times \cos 0$

When the normal to the conductor is at angle $\phi$ to the magnetic field.

When the conductor is parallel to the magnetic field lines $s_{2} \phi=90$.

$\therefore \Phi_{B}=B \times A \times \cos 90$

$=0$ b)

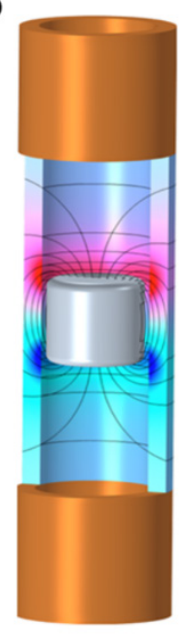

Figure 3. a) Uniform magnetic flux incident on a flat surface at different angles

b) Non-uniform magnetic flux experienced by pipe when a magnet is dropped through it [14]

Since the magnetic flux experienced by copper pipe is not uniform and pipe surface is not flat, see figure $3 \mathrm{~b}$, Equation 1 cannot be directly used for the calculation of the induced emf. After searching Google Scholar for studies on eddy currents induced in copper pipes, using terms such as "fall of magnet through copper pipe", "effect of pipe material on the fall of magnet" I found some interesting research articles that helped me with this problem. One of the articles related the induced eddy currents in pipe with its thickness used quite complicated formulae, which at the time, I was unable to understand [9]. However, this video tutorial [15], which thoroughly explained the mathematical modelling of the eddy currents induced within a pipe by a magnet falling through it, helped me understand the major physical concepts behind my investigation. The full derivation of the formulas used in the report from the video can be found in Appendix a. 
According to the model, a copper pipe can be modelled as many rings of infinitesimally small thickness, and thus the force exerted by the pipe on the magnet can be found by integrating the force exerted by infinitesimally small rings through the dimensions of the pipe, figure 4 .

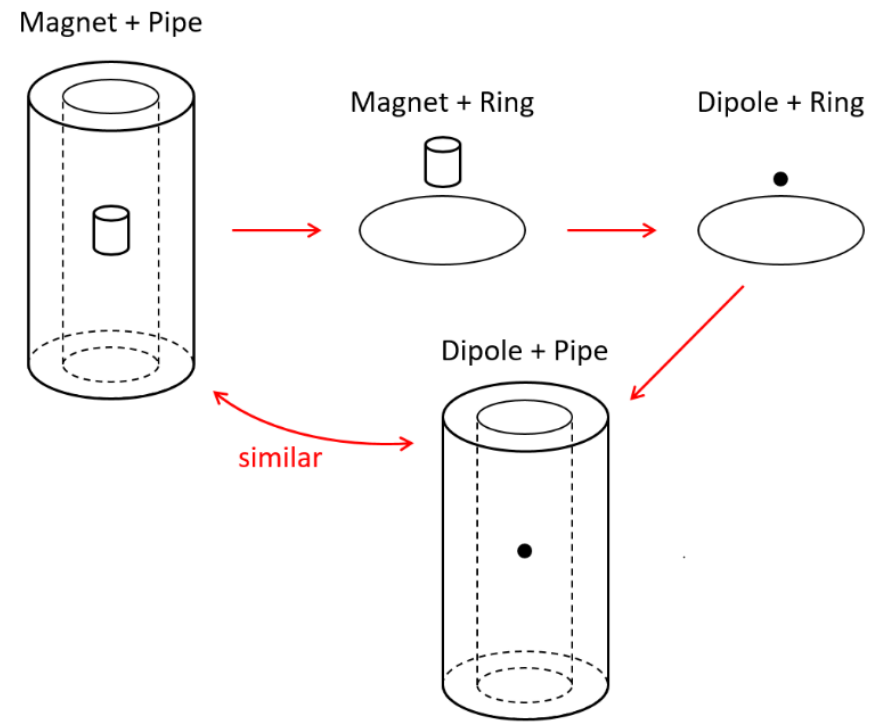

Figure 4. Breaking down the calculation of the retarding force exerted by the pipe on the magnet

The magnetic field of the magnet is assumed equal to the magnetic field $(\vec{B})$ produced by a simple magnetic dipole, given by Equation 3 .

Equation 3:

$$
\vec{B}=\frac{\mu_{0}}{4 \pi} \times\left(\frac{3 \times(\vec{m} \cdot \vec{r}) \times \vec{r}}{r^{5}}-\frac{\vec{m}}{r^{3}}\right)
$$

where $\mu_{0}$ is the magnetic permeability of free space $\left(\mu_{0}=4 \pi \times 10^{-7} N A^{-2}\right), m$ is the magnetic moment of the magnetic dipole, $\vec{r}$ is the position vector of the point experiencing the magnetic field with coordinates $(x, y, z)$.

To determine the magnetic field experienced by the ring, the total magnetic field was split into its $\mathrm{x}, \mathrm{y}$ and $\mathrm{z}$ components. As the pipe is a cylindrical object, it would be more convenient to represent the magnetic field using cylindrical coordinates $(\rho, \phi, \mathrm{z})$ where $\mathrm{z}$ is the vertical displacement of the ring from the magnetic dipole and $\rho$ is the radius of the ring.

\section{Equation 4:}

$$
B_{\rho}=\frac{\mu_{m 0}}{4 \pi} \times \frac{3 z \rho}{\left(\rho^{2}+z^{2}\right)^{\frac{5}{2}}}
$$

\section{Equation 5:}

$$
B_{z}=\frac{\mu_{0} m}{4 \pi} \times\left(\frac{3 \times z^{2}}{\left(\rho^{2}+z^{2}\right)^{\frac{5}{2}}}-\frac{1}{\left(\rho^{2}+z^{2}\right)^{\frac{3}{2}}}\right)
$$

From Equations 4 and 5, the emf induced within the ring can be calculated using Faraday's law of induction [4], resulting in Equation 6. 
Equation 6:

$$
\varepsilon=\frac{3 \mu_{0} m}{2} \times \frac{R^{2} z}{\left(R^{2}+z^{2}\right)^{\frac{5}{2}}} \times v
$$

where $R$ is the outer radius of the pipe, and $v$ is the velocity of the magnet.

Now, with expressions for B_p, Equation 4, and $\varepsilon$, Equation 6, the force exerted by an infinitesimally small ring on the magnet $(\mathrm{dF})$ can be calculated.

Equation 7:

$$
d F=2 \pi R B_{\rho} d I
$$

\section{Equation 8:}

$$
d F=\frac{9 \mu_{0}^{2} m^{2}}{8 \pi} \times v \sigma \times \frac{R^{3} z^{2}}{\left(R^{2}+z^{2}\right)^{5}} d z d R
$$

where $\sigma$ is the conductivity of the pipe.

Using Equation 8, the total force exerted by the pipe on the magnet will be calculated by integrating $d F$ for the dimensions of the pipe. $R$ will be integrated from, the inner radius to the outer radius of the pipe $(a \rightarrow b)$, and $z$ will be integrated from $-\infty$ to $\infty$ to simply the calculations. Importantly, due to the length of the pipe, any change caused by the boundary conditions will have a negligible impact on the experimental results.

$$
F=\int d F=\frac{9 \mu_{0}{ }^{2} m^{2}}{8 \pi} v \sigma \times \int_{a}^{b} \int_{-\infty}^{\infty} \frac{R^{3} z^{2}}{\left(R^{2}+z^{2}\right)^{5}} d z d R
$$

This calculation results in Equation 9.

\section{Equation 9:}

$$
F=\frac{15}{1024} \times \mu_{0}^{2} m^{2} \sigma \times\left(\frac{1}{a^{3}}-\frac{1}{b^{3}}\right) v
$$

Equation 9 is in the same form as Equation 10.

\section{Equation 10:}

$$
F=k v \text {; }
$$

where $k$ is the dragging constant in $\mathrm{kgs}^{-1}$ and hence is given by Equation 11 .

\section{Equation 11:}

$$
k=\frac{15}{1024} \times \mu_{0}^{2} m^{2} \sigma \times\left(\frac{1}{a^{3}}-\frac{1}{b^{3}}\right)
$$

The change in pipe temperature will not change $\mathrm{m}$ nor will it change the thickness of pipe, as the thermal expansion coefficient of copper is very small [16], however the conductivity will decrease significantly. Therefore, the dragging constant $\mathrm{k}$ should decrease with increase in temperature of pipe.

According to Newton's second law [17], the net force on a moving object is $M a=M\left(\frac{d v}{d t}\right)$.

The magnet falling through pipe will experience two opposing forces; a downwards gravitational force (Mg) and an upwards magnetic braking force (F). This leads to Equation 12, which is equivalent to Equation 13.

Equation 12:

$$
M\left(\frac{d v}{d t}\right)=M g-F
$$


Equation 13:

$$
M\left(\frac{d v}{d t}\right)=M g-k v
$$

where $\mathrm{M}$ is the mass of the magnet in $\mathrm{kg}, \mathrm{v}$ is the velocity of the magnet in $\mathrm{ms}^{-1}$ and $\mathrm{g}$ is the gravitational acceleration in $\mathrm{ms}^{-2}$.

When the magnet has reached terminal velocity, $v=v_{T}$, the acceleration is zero $\left(\frac{d v}{d t}=0\right)$, this leads to Equations 14 , 15 and 16.

\section{Equation 14:}

$$
M\left(\frac{d v}{d t}\right)=0=M g-k v_{T}
$$

Equation 15:

$$
M g=k v_{T}
$$

Equation 16:

$$
k=\frac{M g}{v_{T}}
$$

As Neodymium magnets are very strong, when dropped though a copper pipe, they attain terminal velocity within $5 \mathrm{~mm}$. The terminal velocity of the magnet falling through a pipe at different temperatures will be measured practically and inputting this data into Equation 16 will allow experimental $\mathrm{k}$ values to be calculated.

The following section presents the experimental setups used to determine the constants required to calculate the theoretical values of $\mathrm{k}$ and measure terminal velocity of the magnet falling through the pipe at different temperatures.

\section{Laboratory Setup and Experimental Procedure}

In my experiment, the magnet was dropped through a copper pipe, which was held in a vertical position on a retort stand. The motion of the magnet through the copper pipe at various temperatures was monitored using seven pickup coils wrung around the pipe and an oscilloscope. The temperature of the copper pipe was lowered by submerging it in dry ice, and increased with a hair dryer. The finer details of the experiment and procedures are presented in following sections:

\section{Technical Data of Equipment Used}

\section{Cooper Pipe:}

The copper pipe was $150 \mathrm{~cm}$ long with inner diameter $22.4 \pm 0.1 \mathrm{~mm}$ and outer diameter $25.4 \pm 0.1 \mathrm{~mm}$. As preliminary testing showed that the magnet reached terminal velocity within fal, the pipe was cut into a more convenient length of $75 \mathrm{~cm}$.

\section{Magnet:}

A N45 grade neodymium cylindrical magnet with diameter $20 \mathrm{~mm}$, height $25 \mathrm{~mm}$ and weight $71 \mathrm{~g}$ was purchased from AMF Magnetics [18]. The magnet was kept away from other magnetic material to help maintain its magnetic properties. 


\section{Pickup Coils:}

To detect the position of the magnet inside the pipe, seven pick-up coils of $20 \pm 2$ turns made of enamel covered copper wire of thickness $0.3 \mathrm{~mm}$ were wrung around the pipe, see figure 5a. The coils were closely packed onto the pipe, each having a width of $0.5 \mathrm{~cm}$, and were secured to the pipe using both parafilm and electrical tape. The positive ends of the coils and the negative ends were all joined in parallel. Finally, an insulating elastomeric hose was cut and wrapped around the pipe, see figure $5 \mathrm{~b}$, and was fixed in position using electrical tape.
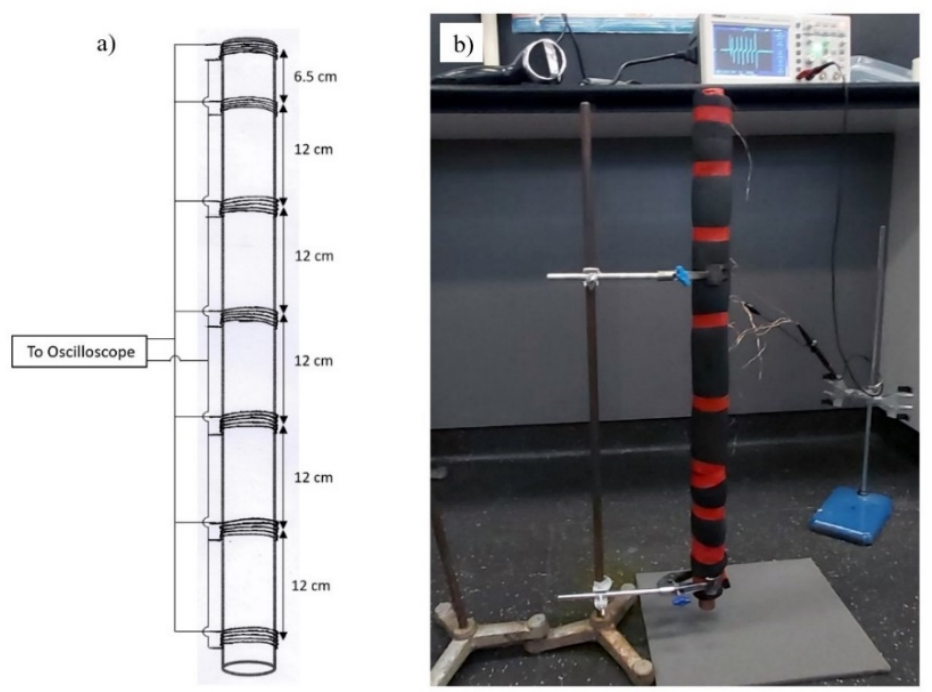

Figure 5. a) Positioning of pick-up coils

b) Experimental setup in laboratory

\section{Oscilloscope:}

An oscilloscope, Tenma 72-10510, was used to measure the induced emf in the pickup coils. The emf vs. time graphs were stored as BITMAP files and processed using the methods described in Section 4c.

\section{Digital infrared thermometer:}

A non-contact, digital infrared thermometer, Ozito IFT-100, working range: $223-373 \mathrm{~K}$, uncertainty: $\pm 2 \mathrm{~K}$, was used to measure the temperature of the copper pipe. A small section of the copper pipe was left exposed at the top and bottom. The thermometer was used to measure the temperature of both sections.

\section{Heating and Cooling the Pipe}

The pipe was cooled to a temperature of about $233 \mathrm{~K}$ using dry ice. This was achieved by filling the pipe with dry ice and placing the filled pipe on a layer of dry ice within a cardboard box, figure 6 . Following this, the pipe was covered with a second layer of dry ice. To heat the pipe, a hair dryer was held above the end of the pipe. This thoroughly heated the air within the pipe, heating the copper pipe uniformly. 


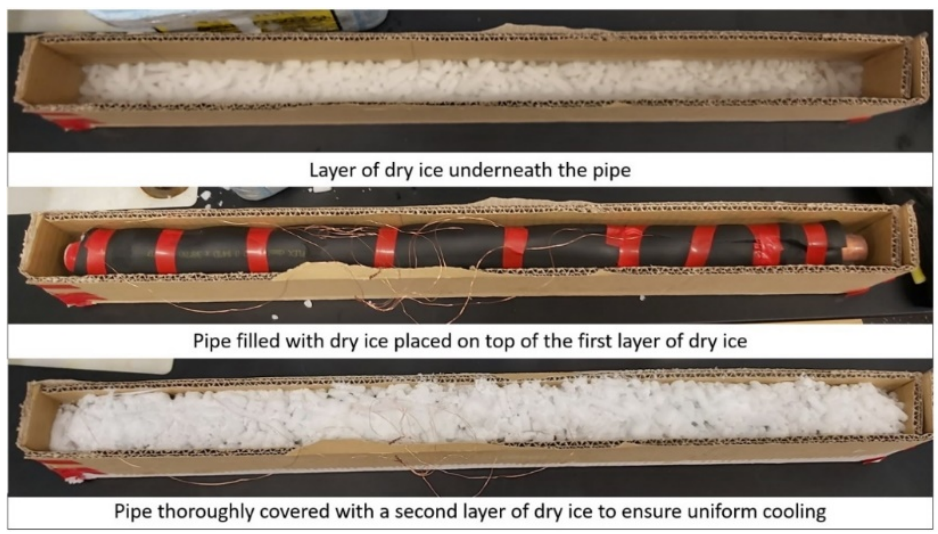

Figure 6. Cooling of the copper pipes with dry ice

\section{c) Experimental Procedure}

The pipe was positioned on a retort stand using two clamps, and was made to be vertical using a spirit level, Figure $5 \mathrm{~b}$. Following this, the clamps were loosened, the pipe was removed from the setup and cooled using dry ice, see Section 2 b. After the pipe reached a temperature of about $233 \mathrm{~K}$, the pipe was promptly removed from the dry ice, taking care to remove the dry ice within the pipe, and clamped back on to the retort stand. Then the two ends of the pickup coils were connected to Channel 1 of the oscilloscope see figure 5a, using two alligator clips and the oscilloscope was switched ON. After waiting 20 minutes for the oscilloscope to warm up, the setup was ready and the trials could begin. The temperature of pipe was measured and recorded. Quickly after measuring the temperature, the oscilloscope's graph was reset and the magnet was dropped down the pipe. Once the magnet exited the pipe, the oscilloscope's graph was paused and a screenshot of the graph was saved onto the attached USB drive with the filename being the temperature of the pipe. A representative graph saved from the oscilloscope is shown in figure 7. Following this, the temperature of the pipe was allowed to rise with the collection of data at regular intervals. Once the pipe was close to room temperature, the pipe was heated to $363 \mathrm{~K}$ and was allowed to cool with the collection of data at regular intervals. This process of conducting trials at regular intervals produced multiple sets of trials with temperatures ranging from 253 to $353 \mathrm{~K}$.

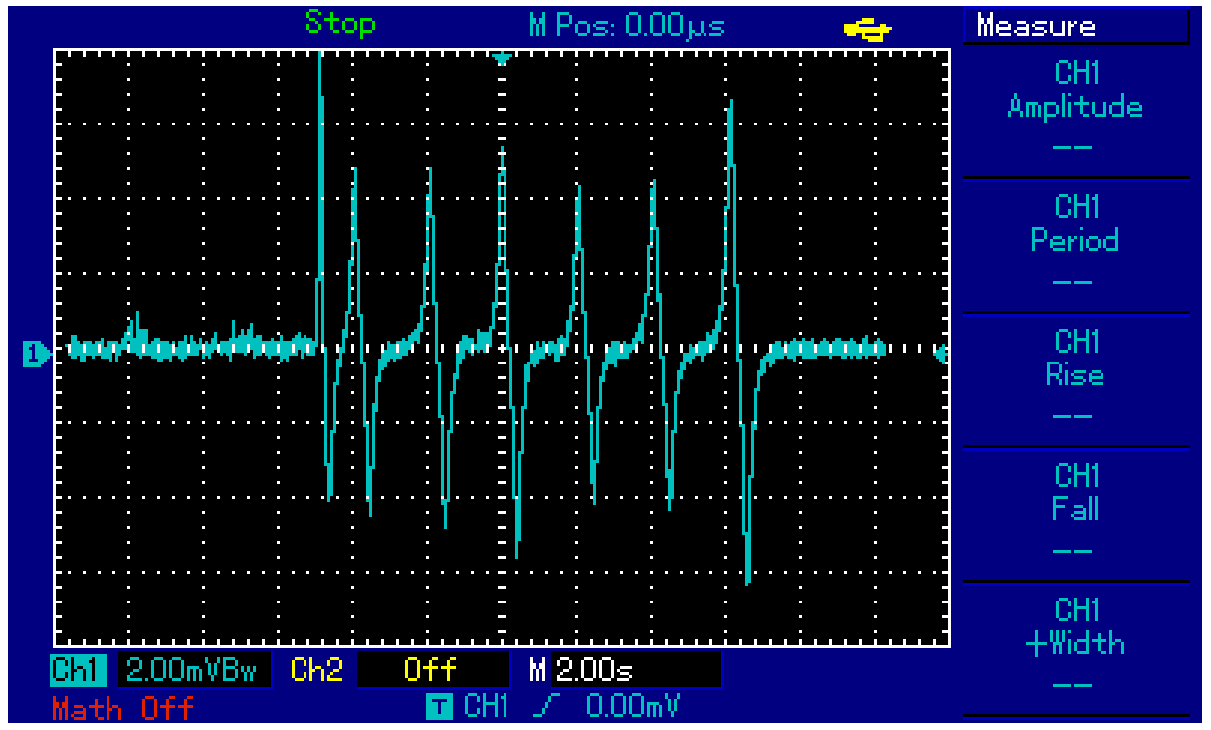

Figure 7. Example of emf vs. time graph produced by the oscilloscope, $T=276 \mathrm{~K}$ 
Data collection, processing and calculations

To calculate the theoretical values of the dragging constant at different temperatures, the magnetic moment of the magnet and the conductivity of the pipe at different temperatures need to be determined.

\section{a) Determining the magnetic moment $(m)$ of the magnet:}

The magnetic moment is a quantity of a magnet that determines the magnitude of force that it can exert on an electric current [19]. The magnetic moment of the magnet used in this study was measured using smartphone with Android® OS as reported in a research paper published in 2015 [20]. A more in-depth explanation of the procedure, data and calculations used to determine $m$ can be found in Appendix b. The final result was $m=7.5 \pm 0.1 \mathrm{Am}^{2}$.

\section{b) Determining the conductivity of the copper pipe $(\sigma)$ at different temperatures}

As detailed in Appendix c and [21], the resistivity of a conductor at different temperatures can be found using Equation 17.

\section{Equation 17:}

$$
\rho_{f}=\rho_{i}\left(1+\alpha\left(T_{f}-T_{i}\right)\right)
$$

where $\rho_{i}$ and $\rho_{f}$ are the resistivity of the conductor at the reference temperature $T_{i}$ and final temperature $T_{f}$ and $\alpha$ is the temperature coefficient of the conductor.

Since $\sigma=\frac{1}{\rho}$ [21], Equation 17 can be used to calculate the conductivity at different temperatures, table 1 .

Table 1. Calculating the conductivity of copper at different temperatures

\begin{tabular}{|c|c|c|c|c|}
\hline $\begin{array}{c}\text { Temperature } \\
\left(T_{F}\right)(\mathrm{K})\end{array}$ & Resistivity $(\rho)(\Omega \mathrm{m})$ & Uncertainty & Conductivity $(\sigma)(\mathrm{S} / \mathrm{m})$ & Uncertainty \\
\hline 253 & $1.41 \times 10^{-8}$ & \multirow{14}{*}{$1.36 \times 10^{-10}$} & $7.10 \times 10^{7}$ & $0.068 \times 10^{7}$ \\
\hline 270 & $1.52 \times 10^{-8}$ & & $6.56 \times 10^{7}$ & $0.058 \times 10^{7}$ \\
\hline 276 & $1.56 \times 10^{-8}$ & & $6.39 \times 10^{7}$ & $0.055 \times 10^{7}$ \\
\hline 283 & $1.61 \times 10^{-8}$ & & $6.20 \times 10^{7}$ & $0.052 \times 10^{7}$ \\
\hline 288 & $1.65 \times 10^{-8}$ & & $6.08 \times 10^{7}$ & $0.050 \times 10^{7}$ \\
\hline 293 & $1.68 \times 10^{-8}$ & & $5.95 \times 10^{7}$ & $0.048 \times 10^{7}$ \\
\hline 303 & $1.75 \times 10^{-8}$ & & $5.72 \times 10^{7}$ & $0.044 \times 10^{7}$ \\
\hline 311 & $1.80 \times 10^{-8}$ & & $5.55 \times 10^{7}$ & $0.042 \times 10^{7}$ \\
\hline 318 & $1.85 \times 10^{-8}$ & & $5.41 \times 10^{7}$ & $0.040 \times 10^{7}$ \\
\hline 323 & $1.88 \times 10^{-8}$ & & $5.31 \times 10^{7}$ & $0.038 \times 10^{7}$ \\
\hline 328 & $1.92 \times 10^{-8}$ & & $5.21 \times 10^{7}$ & $0.037 \times 10^{7}$ \\
\hline 343 & $2.02 \times 10^{-8}$ & & $4.95 \times 10^{7}$ & $0.033 \times 10^{7}$ \\
\hline 348 & $2.05 \times 10^{-8}$ & & $4.87 \times 10^{7}$ & $0.032 \times 10^{7}$ \\
\hline 353 & $2.09 \times 10^{-8}$ & & $4.79 \times 10^{7}$ & $0.031 \times 10^{7}$ \\
\hline
\end{tabular}

Sample calculation of the conductivity of copper at $276 \mathrm{~K}$

According to Wikipedia [21], the resistivity of copper at $293 \mathrm{~K}$ was $1.68 \times 10^{-8} \Omega \mathrm{m}$ and the temperature coefficient was $0.00404 K^{-1}$. Therefore, 


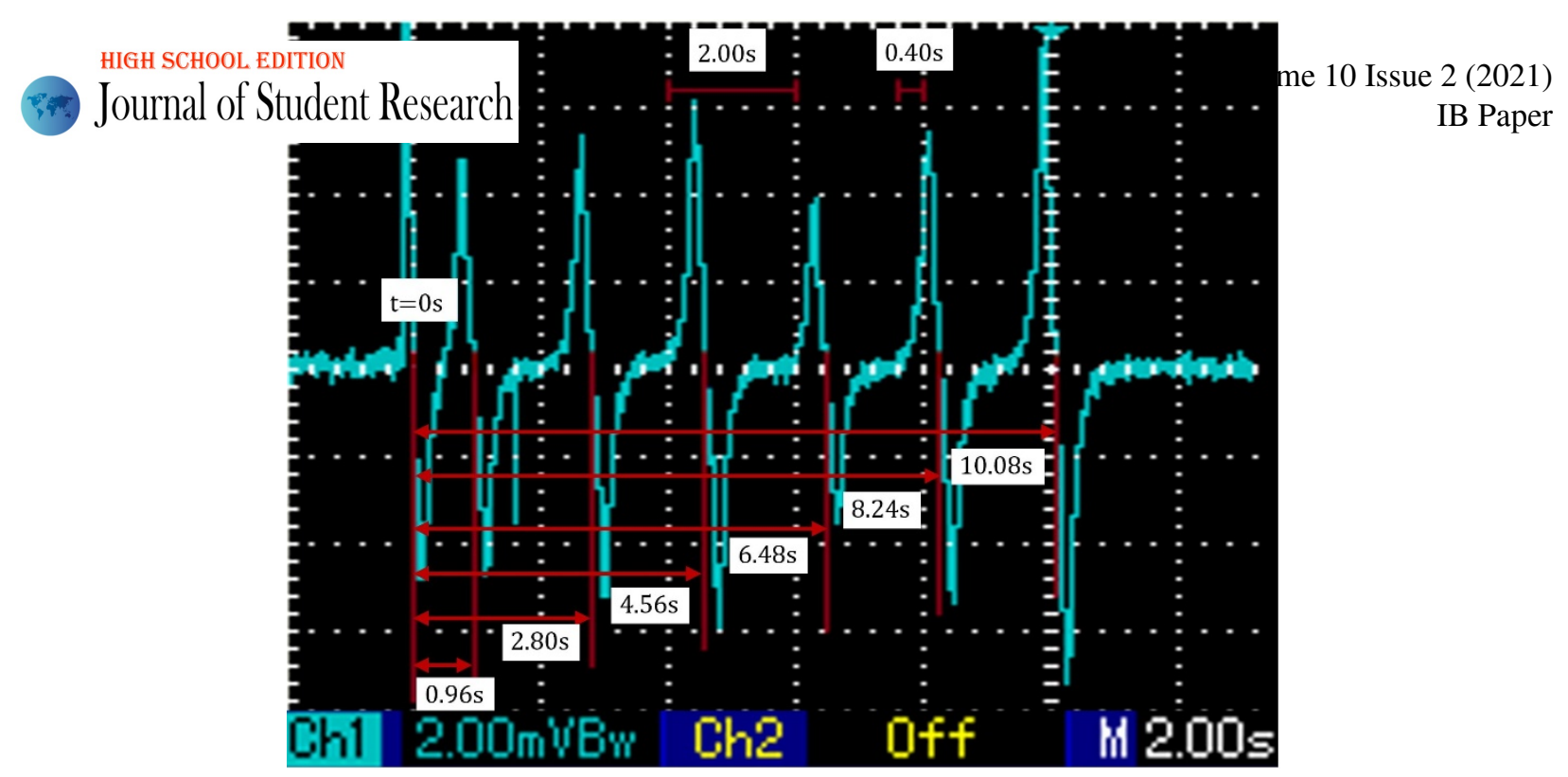

Figure 8. Analysing the voltage vs. time graphs produced by the oscilloscope

$$
\begin{aligned}
\rho_{\text {at } 276 K} & =1.68 \times 10^{-8} \times(1+0.00404((276 \pm 2)-293)) \\
& =1.68 \times 10^{-8} \times(0.93132 \pm 0.00808) \\
& =1.56 \times 10^{-8} \pm 0.0136 \times 10^{-8} \Omega \mathrm{m} \\
\text { Since } \sigma & =\frac{1}{\rho}: \\
\sigma_{\text {at } 276 K} & =\frac{1}{1.56 \times 10^{-8} \pm 0.86 \%} \\
& =6.39 \times 10^{7} \pm 0.055 \times 10^{7} \mathrm{~S} / \mathrm{m}
\end{aligned}
$$

\section{c) Determining the terminal velocity of the magnet at different temperatures}

As described in the Experimental Procedure, the motion of the magnet through the pipe was recorded at temperatures from 253 to $353 \mathrm{~K}$. The oscilloscope graphs were processed to produce displacement vs. time data of the magnet. The displacements vs. time curves were fitted to calculate $v_{T}$ at various temperatures.

Processing of the graphs produced by the oscilloscope:

The voltage versus time BITMAP images produced by the oscilloscope were analysed on a bitmap viewing program, Microsoft Paint. Using this program, the time taken to reach the centre of each coil was determined. The position of the graph where the voltage first rapidly changed from positive to negative as shown by vertical red line, was taken as $t=0$. From this reference point, the number of spaces until the next rapid change was counted and this process was repeated for all six positions, figure 8 . The uncertainty for all times is half of the smallest division and since the oscilloscope has a step size of 0.08 seconds, the uncertainty is \pm 0.04 seconds. This processing procedure produced a table of the time taken by the magnet to reach 7 positions along the pipe, table 2 .

Table 2. Example of data produced from processing of the oscilloscope graphs at $293 \mathrm{~K}$

\begin{tabular}{|c|c|c|c|}
\hline \multirow{2}{*}{ Temperature $(\mathrm{K})$} & Position $(\mathrm{m})( \pm 0.006)$ & \multicolumn{2}{|c|}{ Time taken to reach position $(\mathrm{s})( \pm 0.04)$} \\
\cline { 2 - 4 } & & Trial 1 & Trial 2 \\
\hline \multirow{3}{*}{$293 \pm 2$} & 0.000 & 0.00 & 0.00 \\
\cline { 2 - 4 } & 0.065 & 0.96 & 0.96 \\
\cline { 2 - 4 } & 0.185 & 2.80 & 2.80 \\
\cline { 2 - 4 } & 0.305 & 4.56 & 6.64 \\
\cline { 2 - 4 } & 0.425 & 6.48 & 8.40 \\
\cline { 2 - 4 } & 0.545 & 8.24 & 10.24 \\
\cline { 2 - 4 } & 0.665 & 10.08 & \\
\cline { 2 - 4 } & & & \\
\hline
\end{tabular}


The displacement vs. time data for all trials of each temperature was averaged and the uncertainty in these values was taken to be the standard deviation of the trials. If the standard deviation in the value was below the measured uncertainty of $\pm 0.04 \mathrm{~s}$, the measured uncertainty was used instead. The uncertainty in the displacement of the magnet was taken to be $\pm 0.5 \mathrm{~mm}$ as the uncertainty in the ruler used to position the pickup coils was $\pm 0.5 \mathrm{~mm}$. The full displacement vs. time data is plotted on figure 9, while the data table can be found in Appendix d.
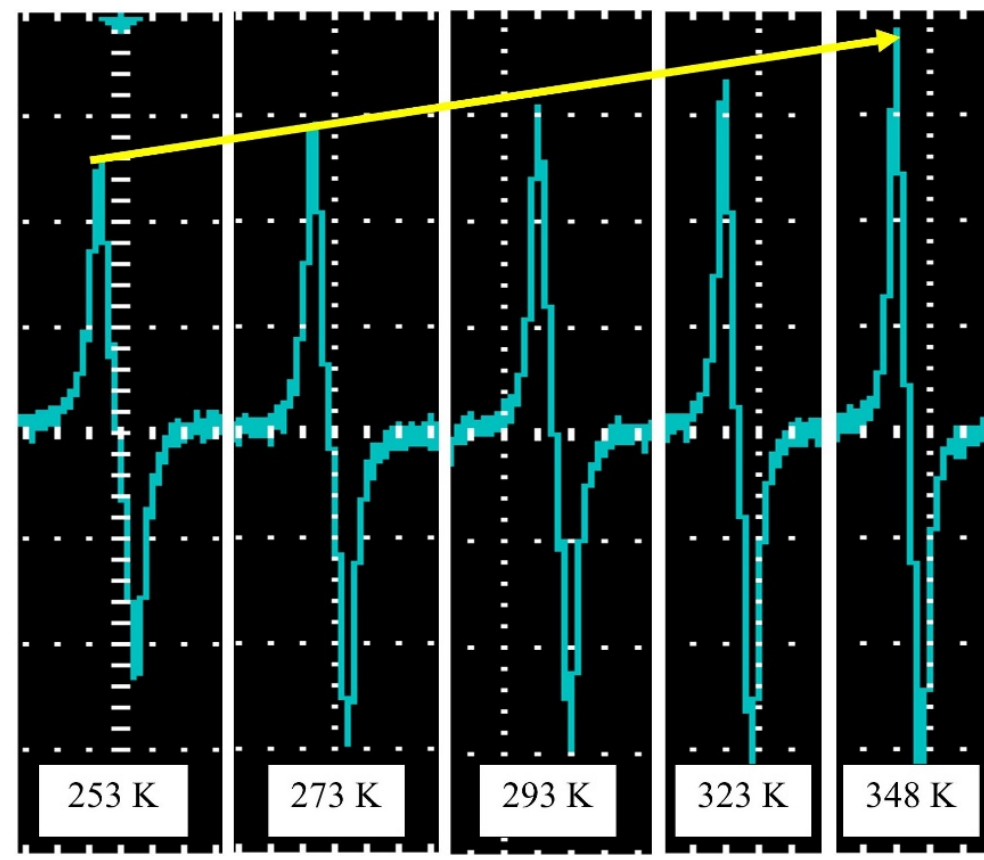

Figure 9. Processed Data - Vertical displacement of magnet vs. time at different temperatures

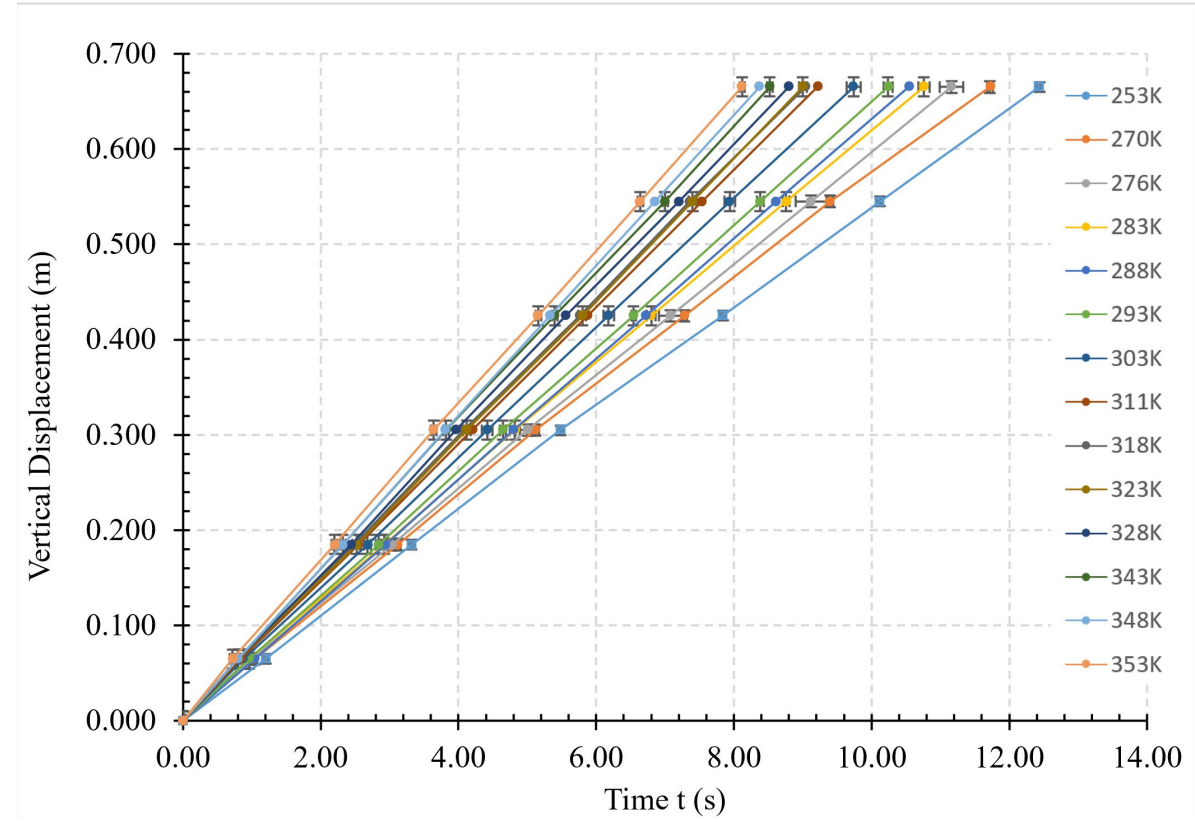

Figure 10. Observation that the emf induced within the coil increased with temperature 
While analysing the graphs produced by the oscilloscope, I observed that as the temperature of the pipe increased, the width of the spikes on the oscilloscope decreased and the height increased. This is in line with my hypothesis as increasing the temperature of the pipe would increase resistivity which would decrease the eddy currents, increasing the terminal velocity. As a result, the rate of change of magnetic flux experienced by the coils was higher and thus the emf induced within the pickup coils was higher, figure 10.

Sample Calculation of Terminal Velocity $v_{T}($ Temperature $=270 \mathrm{~K})$

$v_{T}$ was determined by plotting the displacement of the magnet over time and using a graphing package to find the slope of the best-fit line, see figure 11. The $d=0$ data point was not included during this process, as the magnet had not yet reached terminal velocity.

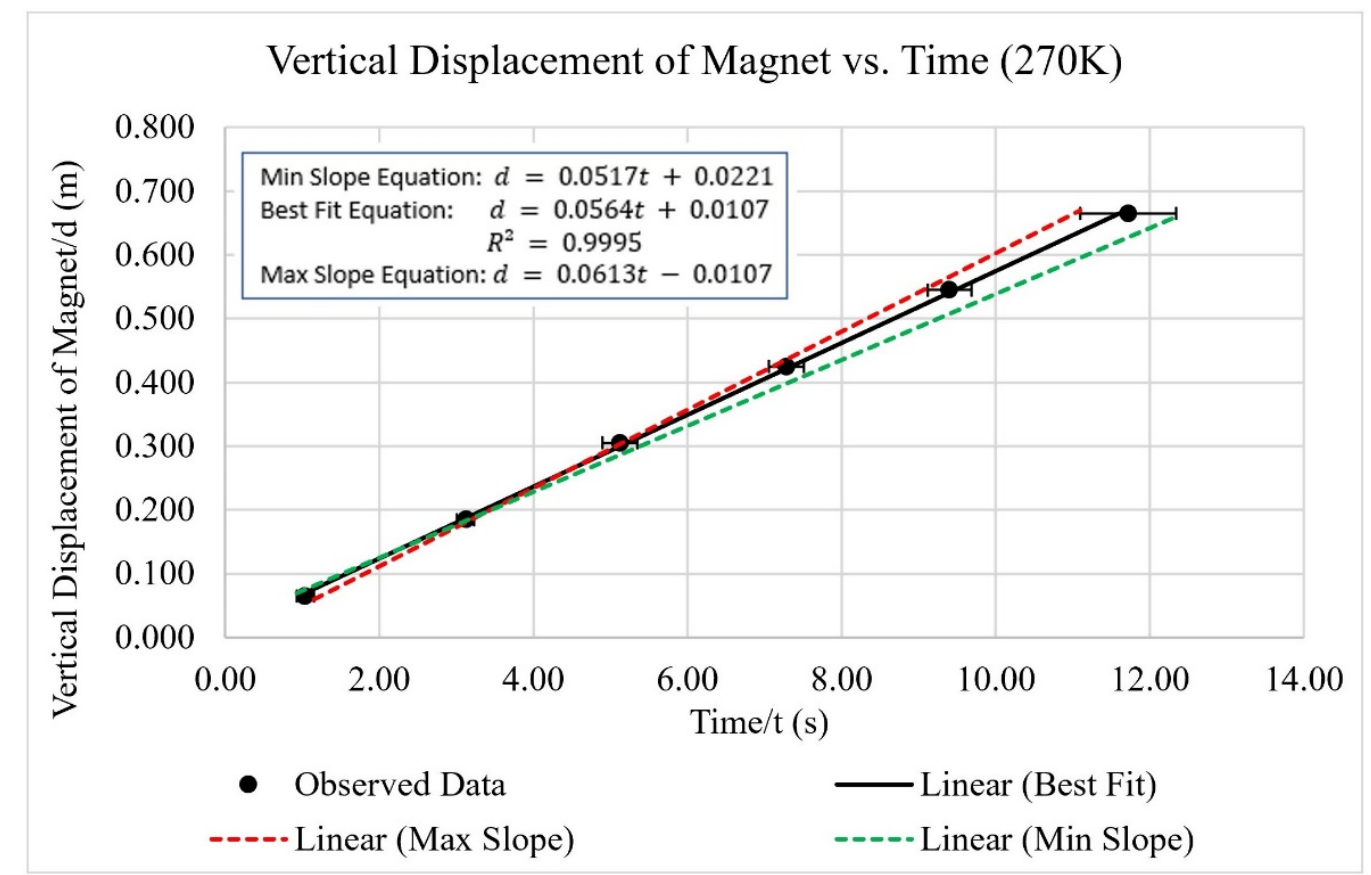

Figure 11. Displacement of magnet vs. time to calculate terminal velocity with max and min trend lines

From figure 11, the terminal velocity of the magnet is $v_{T}=0.0564 \mathrm{~ms}^{-1}$. To find the uncertainty in the experimental value of $v_{T}$, max slope and min slope lines were plotted and used as follows;

uncertainty $\quad=\frac{\max \text { slope }-\min \text { slope }}{2}=\frac{0.0613-0.0517}{2}=0.0048= \pm 0.005 \mathrm{~ms}^{-1}$ (1 s.f.)

$\therefore v_{T}=0.056 \pm 0.005 \mathrm{~ms}^{-1}$ (the place value of the vale of $v_{T}$ must match the place value of its uncertainty).

The full terminal velocity data of the magnet at different temperatures can be seen in table 3 . Interestingly, the displacement vs time best fit lines have very high $\mathrm{R}^{2}$ values, indicating that the variation in $v_{T}$ within trials at one temperature is very small and that the velocity of the magnet through the pipe is constant, hence the temperature and other physical properties throughout the pipe were uniform. 
Table 3. Terminal velocity of magnet at various temperatures

\begin{tabular}{|l|l|l|l|l|}
\hline $\begin{array}{l}\text { Temperature } \\
(\mathrm{T}) \pm 2(\mathrm{~K})\end{array}$ & $\begin{array}{l}\text { No. of tri- } \\
\text { als }(\mathrm{n})\end{array}$ & Equation of Line of Best Fit & $\begin{array}{l}\text { Correlation Coef- } \\
\text { ficient }\left(\mathrm{r}^{2}\right)\end{array}$ & Terminal Velocity $\left(\mathrm{v}_{\mathrm{T}}\right)\left(\mathrm{ms}^{-1}\right)$ \\
\hline 253 & 2 & $\mathrm{~d}=0.0532 \mathrm{t}+0.007$ & 0.9996 & $0.053 \pm 0.001$ \\
\hline 270 & 2 & $\mathrm{~d}=0.0564 \mathrm{t}+0.0107$ & 0.9995 & $0.056 \pm 0.005$ \\
\hline 276 & 2 & $\mathrm{~d}=0.0591 \mathrm{t}+0.0067$ & 0.9999 & $0.059 \pm 0.002$ \\
\hline 283 & 4 & $\mathrm{~d}=0.0613 \mathrm{t}+0.0071$ & 0.9999 & $0.061 \pm 0.002$ \\
\hline 288 & 9 & $\mathrm{~d}=0.0632 \mathrm{t}-0.0001$ & 0.9999 & $0.063 \pm 0.002$ \\
\hline 293 & 10 & $\mathrm{~d}=0.0647 \mathrm{t}+0.0026$ & 0.9999 & $0.065 \pm 0.002$ \\
\hline 303 & 4 & $\mathrm{~d}=0.0679 \mathrm{t}+0.0046$ & 0.9999 & $0.068 \pm 0.002$ \\
\hline 311 & 3 & $\mathrm{~d}=0.0718 \mathrm{t}+0.0035$ & 0.9999 & $0.072 \pm 0.003$ \\
\hline 318 & 2 & $\mathrm{~d}=0.073 \mathrm{t}+0.0058$ & 0.9999 & $0.073 \pm 0.002$ \\
\hline 323 & 2 & $\mathrm{~d}=0.0733 \mathrm{t}+0.0031$ & 0.9999 & $0.073 \pm 0.002$ \\
\hline 328 & 2 & $\mathrm{~d}=0.0752 \mathrm{t}+0.0048$ & 0.9999 & $0.075 \pm 0.003$ \\
\hline 343 & 2 & $\mathrm{~d}=0.0772 \mathrm{t}+0.0062$ & 0.9999 & $0.077 \pm 0.003$ \\
\hline 348 & 3 & $\mathrm{~d}=0.0793 \mathrm{t}+0.0018$ & 0.9999 & $0.079 \pm 0.003$ \\
\hline 353 & 2 & $\mathrm{~d}=0.081 \mathrm{t}+0.0075$ & 0.9999 & $0.081 \pm 0.002$ \\
\hline
\end{tabular}

d) Calculating the theoretical and experimental dragging constants at different temperatures

Sample Calculation of Theoretical $\mathrm{k}$ at $\mathrm{T}=276 \mathrm{~K}$

The theoretical values for $\mathrm{k}$ will be calculated using Equation 11.

\section{Equation 11:}

$$
\begin{aligned}
& \quad k=\frac{15}{1024} \times \mu_{0}{ }^{2} m^{2} \sigma \times\left(\frac{1}{a^{3}}-\frac{1}{b^{3}}\right) \\
& \therefore \mathrm{k}_{\text {at } 276 \mathrm{~K}}=\frac{15 \times\left(4 \pi \times 10^{-7}\right)^{2} \times(7.5 \pm 0.1)^{2} \times(6.39 \pm 0.055) \times 10^{7}}{1024} \times\left(\frac{1}{\left((11.2 \pm 0.05) \times 10^{-3}\right)^{3}}-\frac{1}{\left((12.7 \pm 0.05) \times 10^{-3}\right)^{3}}\right) \\
&=8.31 \times 10^{-5} \pm\left(2 \times \frac{0.1}{7.5} \times 100 \%+\frac{0.055}{6.39} \times 100 \%\right) \times\left(7.12 \times 10^{5} \pm\left(3 \times \frac{0.05}{11.2} \times 100 \%\right)-4.88 \times\right. \\
&\left.10^{5} \pm\left(3 \times \frac{0.05}{12.7} \times 100 \%\right)\right) \\
&=8.31 \times 10^{-5} \pm(3.527 \%) \times\left(7.12 \times 10^{5} \pm(1.339 \%)-4.88 \times 10^{5} \pm(1.181 \%)\right) \\
&= 8.31 \times 10^{-5} \pm(3.527 \%) \times\left(2.24 \times 10^{5} \pm\left(0.2 \times 10^{5}\right)\right) \\
&=18.68 \pm\left(3.527 \%+\frac{0.2 \times 10^{5}}{2.24 \times 10^{5}} \times 100 \%\right) \\
&=18.68 \pm(10.36 \%) \\
&=18.68 \pm(1.94) \mathrm{kgs}^{-1} \\
& \therefore \mathrm{k}_{\text {at } 276 \mathrm{~K}}=19 \pm 2 \mathrm{kgs}^{-1}
\end{aligned}
$$

Sample Calculation of Experimental k at $\mathrm{T}=276 \mathrm{~K}$

The experimental values for $\mathrm{k}$ will be calculated using Equation 16.

\section{Equation 16:}

$\therefore k_{\text {at } 276 K}=\frac{0.071 \times 9.81}{0.059 \pm 0.002}$

$$
k=\frac{M g}{v_{T}}
$$

$=11.81 \pm\left(\frac{0.002}{0.059} \times 100 \%\right)$

$\therefore k_{\text {at } 276 \mathrm{~K}}=11.8 \pm 0.4 \mathrm{kgs}^{-1}$ 
The theoretical and experimental dragging constants at each temperature are presented in table 4 and are plotted in figure 12. The error bars shown on figure 12 represent the absolute uncertainty in $\mathrm{k}$ and $\mathrm{T}$.

Table 4. Experimental and theoretical $\mathrm{k}$ at temperatures ranging from 253 to $353 \mathrm{~K}$

\begin{tabular}{|c|c|c|}
\hline Temperature $\mathrm{T}(\mathrm{K})( \pm 2)$ & $\begin{array}{c}\text { Experimental } \mathrm{k} \\
\left(\mathrm{kgs}^{-1}\right)\end{array}$ & $\begin{array}{c}\text { Theoretical k } \\
\left(\mathrm{kgs}^{-1}\right)\end{array}$ \\
\hline 253 & $13.1 \pm 0.2$ & $21 \pm 2$ \\
\hline 270 & $12 \pm 1$ & $19 \pm 2$ \\
\hline 276 & $11.8 \pm 0.4$ & $19 \pm 2$ \\
\hline 283 & $11.4 \pm 0.4$ & $18 \pm 2$ \\
\hline 288 & $11.1 \pm 0.4$ & $18 \pm 2$ \\
\hline 293 & $10.7 \pm 0.3$ & $17 \pm 2$ \\
\hline 303 & $10.2 \pm 0.3$ & $17 \pm 2$ \\
\hline 311 & $9.7 \pm 0.4$ & $16 \pm 2$ \\
\hline 318 & $9.5 \pm 0.3$ & $16 \pm 2$ \\
\hline 323 & $9.5 \pm 0.3$ & $16 \pm 2$ \\
\hline 328 & $9.3 \pm 0.4$ & $15 \pm 2$ \\
\hline 343 & $9.0 \pm 0.4$ & $14 \pm 1$ \\
\hline 348 & $8.8 \pm 0.3$ & $14 \pm 1$ \\
\hline 353 & $8.6 \pm 0.2$ & $14 \pm 1$ \\
\hline
\end{tabular}

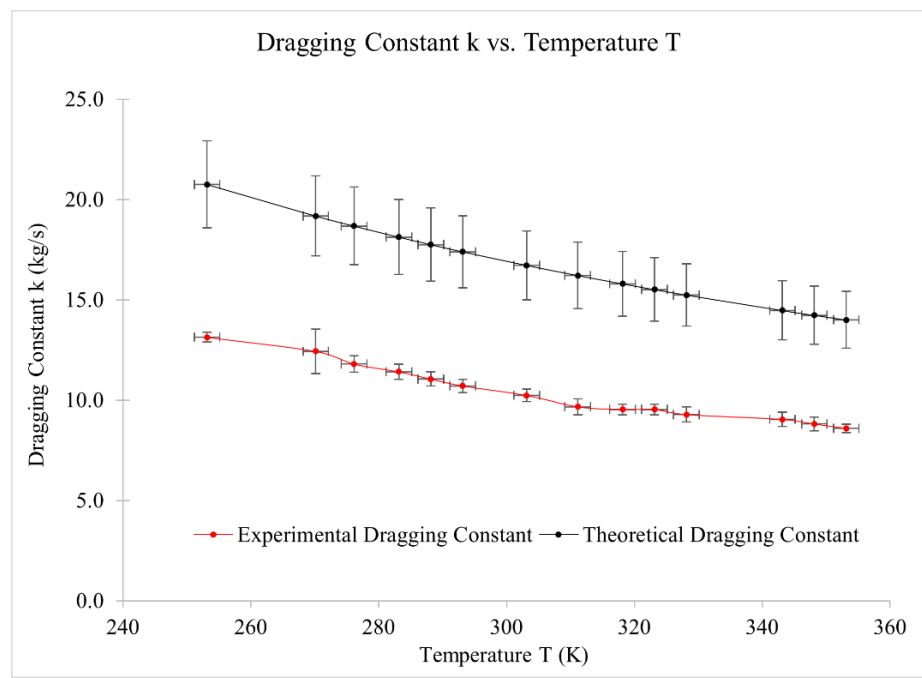

Figure 12. Experimental and theoretical dragging constant vs. temperature of copper pipe

\section{Evaluation}

As tabulated in table 3, the terminal velocity of the magnet increases as temperature of the copper pipe it is falling through increases, supporting my hypothesis that an increase in the pipe's temperature will increase its resistance, leading to a decrease in eddy current flow and hence reducing the retarding force exerted by the pipe on the magnet. From figure 12, it is evident that both the theoretically calculated and the experimentally measured $k$ follow a very similar trend; both decrease with an increase in the pipe's temperature, supporting the hypothesis that $\mathrm{k}$ depends on the pipe's resistance. Moreover, the mathematical model used successfully models the effect of pipe temperature on 
$k$ and thus enables the prediction of the retarding force experienced by a magnet falling through a pipe at different temperatures. However, figure 12 also shows that the experimentally calculated $\mathrm{k}$ is much lower than the theoretical $\mathrm{k}$, indicating significant systematic error. Also, the experimentally observed data forms a quite smooth line with little zagging, indicating minimal random error.

\section{a) Systematic Errors}

It is clear from Equation 11 that the dragging constant $(k)$ is dependent on $m, \frac{1}{\mathrm{a}^{3}}-\frac{1}{\mathrm{~b}^{3}}$ and $\sigma$. It follows that the large systematic error should be a result of errors and inaccuracies in one or combination of these three variables.

\section{i. The magnetic moment $(m)$}

From equation $11, k \propto m^{2}$. As a result, inaccuracies in the experimentally measured magnetic moment will have a large impact on the theoretical dragging constants. In this report, the magnetic moment of the magnet was determined using a procedure described in Section $4 \mathrm{a}$ and Appendix $\mathrm{b}$. The experimentally calculated magnetic moment was $7.5 \mathrm{Am}^{2}$, which is similar to the value calculated from its residual flux density. As a result, this cannot be the sole cause of the large systematic error. In addition, it was observed that magnet warmed up considerably when moving through the hot pipe, however this should not have impacted its magnetic properties as N45 magnets have a working temperature of $\leq 353 \mathrm{~K}$ [22].

\section{ii. The inner and outer radiuses of the pipe $\left(\frac{1}{a^{3}}-\frac{1}{b^{3}}\right)$}

From Equation 11, $k \propto\left(\frac{1}{\mathrm{a}^{3}}-\frac{1}{\mathrm{~b}^{3}}\right)$. As a result, increases in the outer radius (b) and decreases in the inner radius (a) of the pipe will increase the theoretical dragging constant. The copper coils that were wrung around the pipe would slightly increase the outer radius, and a result the theoretical dragging constants should be slightly higher. Moreover, when heated copper expands slightly [16], which would decrease the inner radius and increase the outer radius. Together both of these errors would lead to a slight increase in $\left(\frac{1}{\mathrm{a}^{3}}-\frac{1}{\mathrm{~b}^{3}}\right)$, which in turn means that the theoretical dragging constants are a little smaller than what they should be. However, since the magnitude of this error is so small, it is negligible.

\section{iii. The conductivity of the pipe $(\sigma)$}

From equation $11, k \propto \sigma$. The conductivity of a copper pipe decreases with the presence of impurities, and the small amounts of phosphorus present within the pipe [23] will significantly decrease (by $\approx 35 \%$ ) its conductivity [24, Fig. 1]. This large decrease in conductivity would cause a significant decrease in the theoretical dragging constants, significantly reducing the gap between the theoretical and experiment dragging constants.

Equation 11 can be written as Equation 18.

\section{Equation 18:}

$$
k=k^{\prime} \sigma
$$

where $k^{\prime}$ is a constant equal to $\frac{15}{1024} \times \mu_{0}{ }^{2} m^{2} \times\left(\frac{1}{a^{3}}-\frac{1}{b^{3}}\right)$

This leads to Equation 19.

\section{Equation 19:}

$$
\therefore \frac{1}{k}=\frac{1}{k^{\prime}} \times \frac{1}{\sigma}
$$

From Equation 17, the inverse of the conductivity at different temperatures can be expressed as the following equation. 


\section{Equation 20:}

Leading to the following equation.

$$
\begin{gathered}
\frac{1}{\sigma_{f}}=\rho_{i}+\alpha \rho_{i} \Delta T \\
\therefore \frac{1}{k}=\frac{1}{k^{\prime}} \times\left(\rho_{i}+\alpha \rho_{i} \Delta T\right)
\end{gathered}
$$

\section{Equation 21:}

$$
\therefore \frac{1}{k}=\frac{\rho_{i}}{k^{\prime}}+\frac{\alpha \rho_{i}}{k^{\prime}} \times \Delta T
$$

From Equation 21, we can deduce that $\frac{1}{k}$ will linearly change with temperature. As a result graphing $\frac{1}{k}$ against $\mathrm{T}$ will facilitate quantitative analysis of the impact of decreasing the conductivity. The theoretical $\mathrm{k}$ values at different temperatures, considering the conductivity of pipe is $65 \%$ of a pipe made up of pure copper, were calculated and along with the experimental $\mathrm{k}$ values were inversed and plotted against temperature with appropriate error bars, figure 13. The equations and correlation coefficients of the best-fit lines are presented in table 5.

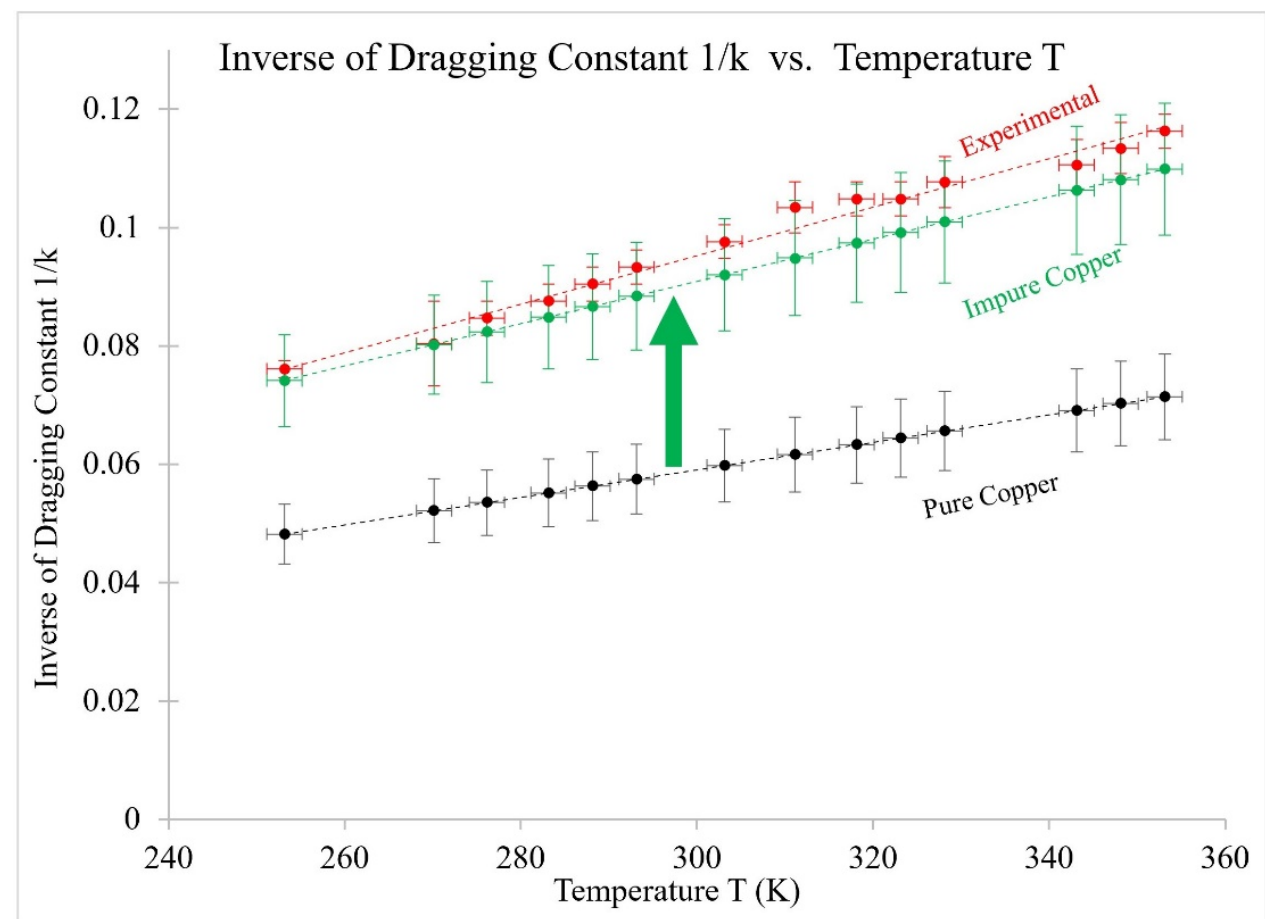

Figure 13. Inverse of dragging constant vs. temperature of copper pipe

Table 5. Inverse of dragging constant vs. temperature line of best fit equations

\begin{tabular}{|c|c|c|}
\hline & Equation of Line of Best Fit & $\begin{array}{c}\text { Correlation } \\
\text { Coefficient }\left(r^{2}\right)\end{array}$ \\
\hline Experimental & $\frac{1}{k}=0.00041 T-0.02752$ & 0.98259 \\
\hline Theoretical (Impure copper) & $\frac{1}{k}=0.00036 T-0.01630$ & 1.00000 \\
\hline Theoretical (Pure copper) & $\frac{1}{k}=0.00023 T-0.01059$ & 1.00000 \\
\hline
\end{tabular}


Looking at figure 13 , the experimental $\mathrm{k}$ data is within the uncertainty range of the theoretical $\mathrm{k}$ data that takes the reduced conductivity into account. Moreover the slope of the experimental data 0.00041 is very similar to that of the theoretical data that takes the reduced conductivity into account 0.00036 .

\section{b) Random Errors}

As discussed earleir, the small amount of zagging in the observed k vs. temperature data indicates the presence of small random error, which is further supported by high $\mathrm{R}^{2}$ value $(0.98259)$ of the $\frac{1}{k}$ vs. temperature data linear best fit, figure 13. The possible source of random errors and their impact on the results are discussed below:

\section{i. Imprecise thermometer}

The thermometer used had low precision, and this along with the high rate of temperature change (especially at temperatures below $273 \mathrm{~K}$ and above $323 \mathrm{~K}$ ) resulted in a small number of trials for some of the temperature ranges, leading to some random error.

\section{ii. Water droplets within the pipe}

Water droplets present within the pipe when cooled to low temperatures, due to the humidity of the laboratory, may have small impact on the terminal velocity of the magnet. However, due to the apparent lack of discontinuities between the data at low and high temperatures it may be concluded that the impact of water droplets within the pipe is negligible.

\section{iii. Position of the copper pipe}

It is possible that the position of copper pipe may have changed slightly in-between trials, and hence was not perfectly vertical in some trials. The terminal velocity in those trials will be slightly lower than expected, in turn leading to unexpected increases in the value of the dragging constant in some trials.

\section{c) Improvements and Extensions}

\section{i. Using a copper pipe with known conductivity:}

As discussed in Section 5a. this investigation's major flaw is the unknown conductivity of the copper pipe used. This flaw is the most probable reason for the large systematic error. Using a copper pipe with known conductivity will eliminate this systematic error, ensuring the validity of the mathematical model used, and hence allowing the hypothesis to be tested more rigourously.

\section{ii. Using a magnetometer to accurately measure the magnetic field}

As discussed in Section 5a. a possible cause of the significant systematic error observed in Figure 12 is inaccuracy in the value of $m$. Using a magnetometer rather than a smartphone will improve the accuracy of the magnetic field measurements, improving the accuracy of $m$, and hence will reduce the systematic error.

\section{iii. Greater number of trials at low and high temperatures}

As evident from Table 3, for many of the temperature ranges only two trials were performed. Increasing the number of trials will limit the impact of random errors, increasing the precision of the experimental data. 


\section{Conclusion}

The equipment readily available in the school laboratory and materials available from local retailers were successfully used to understand the effect of temperature on eddy currents. This study has clearly established that the terminal velocity of magnet falling through a copper pipe is proportional to pipe's temperature.

In addition, a literature model of the retarding force exerted on a magnet falling through a conductive pipe was used to quantitatively explain the change in dragging constant with temperature. However, its success was limited due to the presence of large systematic error, possibly caused by impurities in the copper pipe that would have significantly reduced its conductivity. As a result, further scientific study is needed to assess the accuracy of the mathematical model.

Ultimately, this study establishes that temperature, i.e. the variable weather around the globe, plays a significant role and needs to be considered when designing eddy current based machines. such as magnetic brakes in highspeed trains and maglev transportation. More specifically, it experimentally shows that in the temperature range - 20 to $80^{\circ} \mathrm{C}$ the drag force caused by eddy currents varies from 13.1 to $8.6 \mathrm{kgs}^{-1}$. Consequently, eddy current based machines in some of the coldest regions of the world, such as Canada, will be far more efficient than if they were used in the hottest regions of the world, for instance Burkina Faso. Importantly however, actual linear eddy current brakes utilise the rail which is often made of stainless steel a material that has a considerably lower thermal and electrical conductivity which will reduce the impact of temperature on the drag force caused by the linear eddy current brake.

\section{Acknowledgments}

I would like to thank my advisor John Duivestein for helping me with this project.

\section{References}

[1] The Editors of Encyclopædia Britannica, Eddy current, Encyclopædia Britannica inc. May 1, 2017. Accessed on: Nov. 27, 2019. [Online]. Available: https://www.britannica.com/science/eddy-current

[2] Wouterse J. Critical torque and speed of eddy current brake with widely separated soft iron poles. IEE Proc-B. 1991;138:153-158

[3] Wikipedia contributors, Eddy current brake, Wikipedia, The Free Encyclopedia. Mar. 3, 2020. Accessed on: Nov. 2, 2019. [Online]. Available: https://en.wikipedia.org/wiki/Eddy current brake

[4] The Editors of Encyclopædia Britannica, Faraday's law of induction, Encyclopædia Britannica inc. Sept. 6, 2013. Accessed on: Nov. 27, 2019. [Online]. Available: https://www.britannica.com/science/Faradays-law-of-induction

[5] The Editors of Encyclopædia Britannica, Lenz's law, Encyclopædia Britannica inc. Jan. 4, 2018. Accessed on: Nov. 27, 2019. [Online]. Available: https://www.britannica.com/science/Lenzs-law

[6] D. Homer and M. Bowen-Jones, Physics Course Companion, 2014 ed. Oxford, England: Oxford University Press, 2014. 
[7] C. L. Ladera, G. Donoso, and P. Martín, "One or two magnets falling in a conductive pipe: On-axis and off-axis fall and the role of the pipe wall thickness," Lat. Am. J. Phys. Educ, vol. 6, suppl. I, pp. 216-221, Aug. 2012. Accessed on: Dec. 1, 2019. [Online]. Available: http://www.lajpe.org/icpe2011/40 Celso Ladera.pdf

[8] B. Rizzato, F. L. da Silveira and Y. Levin, "Electromagnetic braking: A simple quantitative model," Am. J. Phys. vol. 74, no. 9, pp. 815-817, Dec. 1, 2019. Accessed on: May 7, 2020. [Online]. Available: http://www.if.ufrgs.br/ levin/Pdfs.dir/AJP000815.pdf

[9] C. L. Ladera, G. Donoso, and P. Martín, "Magnet fall inside a conductive pipe: motion and the role of the pipe wall thickness," Eu. J. Phys. vol. 30, no. 4, pp. 855-869, May 2009. Accessed on: Dec. 1, 2019. [Online]. Available: https://pdfs.semanticscholar.org/0057/1f4604107499b4bb1713a2d4822b7a315631.pdf

[10] F. G. Tomasel and M. C. Marconi, Rolling magnets down a conductive hill: Revisiting a classic demonstration of the effects of eddy currents, Am. J. Phys. vol. 80, no. 9, pp. 800-803, Sept. 2012. Accessed on: Aug. 7, 2019. [Online]. Available: http://www.msc.univ-parisdiderot.fr/ phyexp/uploads/LaimantParesseux/aimant2.pdf

[11] K. Akhtar, The relationship between electrical conductivity and magnetically damped motion, Queensland Academy for Science, Mathematics and Technology. Accessed on: Aug. 7, 2019. [Online]. Available: $\underline{\text { https://digitalcommons.imsa.edu/cgi/viewcontent.cgi?article=1089\&context=issf2018 }}$

[12] Wikipedia contributors, Temperature coefficient, Wikipedia, The Free Encyclopedia. Mar. 1, 2020. Accessed on: Dec. 5, 2019. [Online]. Available: https://en.wikipedia.org/w/index.php?title=Temperature coefficient\&oldid $=943452936$

[13] The Physics Hypertextbook. 2021. Electric Resistance. [ONLINE] Available at: https://physics.info/electric-resistance/. [Accessed 22 May 2021].

[14] T. Forrister, How Eddy Current Braking Technology Is Freeing Us from Friction, COMSOL Blog, Mar. 2019. Accessed on: Nov. 2, 2019. [Online]. Available: https://www.comsol.com/blogs/howeddy-current-braking-technology-is-freeing-us-from-friction/

[15] L. Hanson, Northeastern IPL, Physics in Action: Magnet Falling Through a Conductive Pipe, May 16, 2017. [Video file]. Available: https://www.youtube.com/watch?v=2-iEVFICIqM 
[16] Wikipedia contributors, Thermal expansion, Wikipedia, The Free Encyclopedia. May 6, 2020. Accessed on: Dec. 5, 2019. [Online]. Available: https://en.wikipedia.org/w/index.php?title=Thermal expansion\&oldid $=955208327$

[17] The Editors of Encyclopædia Britannica, Newton's laws of motion, Encyclopædia Britannica inc. Feb. 3, 2020. Accessed on: Nov. 27, 2019. [Online]. Available: https://www.britannica.com/science/Newtons-laws-of-motion

[18] “Rare Earth (Neodymium) Cylinder Magnets,” AMF Magnetics, 2020. Accessed on: Aug. 5, 2019. [Online]. Available: https://magnet.com.au/rare-earth-magnets-neodymium-cylinders.html

[19] J. Tatum, The SI Definition of Magnetic Moment, Physics LibreTexts. Jun. 3, 2019. Accessed on: Nov. 27, 2019. [Online]. Available: https://phys.libretexts.org/Bookshelves/Electricity and Magnetism/Book\%3A Electricity and Magnetism (Tatum)/17\%3A Magnetic Dipole Moment/17.02\%3A The SI Definition of Magnetic Moment

[20] A. Nájera, A. Beléndez, C. P. Suárez, E. Arribas and I. Escobar "Measurement of the magnetic field of small magnets with a smartphone: a very economical laboratory practice for introductory physics courses," Eu. J. Phys. vol. 36, no. 6, pp. 1-12, Aug. 2015. Accessed on: Dec. 1, 2019. [Online]. Available: https://pdfs.semanticscholar.org/6fdd/153d69bcb9c00587e3926903cfed90c897c9.pdf

[21] Wikipedia contributors, Electrical resistivity and conductivity, Wikipedia, The Free Encyclopedia. May 5, 2020. Accessed on: Nov. 23, 2019. [Online]. Available: https://en.wikipedia.org/w/index.php?title=Electrical resistivity and conductivity\&oldid $=954934185$

[22] “Typical Magnetic Properties for Rare Earth Magnets," AMF Magnetics, 2020. Accessed on: Feb. 5, 2020. [Online]. Available: https://amfmagnets.com/typical-magnetic-properties-for-rare-earth-magnets.html

[23] The Plumbers Handbook, 9th ed. International Copper Association Australia, Copper Alliance, Australia, Mar. 2016, p. 10. Accessed on: Dec. 5, 2019. [Online]. Available: https://www.kembla.com/assets/Uploads/general-PDFs/The-Plumbers-Handbook-9th-Edition.pdf

[24] D. Chapman, High Conductivity Copper for Electrical Engineering, Copper Development Association, Copper Alliance, Dec. 5, 2019. Accessed on: May 7, 2020. [Online]. Availa-

ble: http://copperalliance.org.uk/uploads/2018/02/pub-122-hicon-copper-for-electrical-engineering.pdf

\section{Appendix}

a) Full derivation of the formulas taken from the YouTube video [13]

Expression for the magnetic field of a magnetic dipole: 
Equation A1:

$$
\vec{B}=\frac{\mu_{0}}{4 \pi} \times\left(\frac{3 \times(\vec{m} \cdot \vec{r}) \times \vec{r}}{r^{5}}-\frac{\vec{m}}{r^{3}}\right) ;
$$

where $\mu_{0}$ is the magnetic permeability of free space, $m$ is the magnetic moment and $\vec{r}$ is an arbitrary vector representing the position experiencing the magnetic field with respect to the position of the magnetic dipole with cords $(x, y$, z) $\therefore \mathrm{r}=\sqrt{\mathrm{x}^{2}+\mathrm{y}^{2}+\mathrm{z}^{2}}$

We have chosen $\vec{m}$ to be in the $z$ direction, figure a.

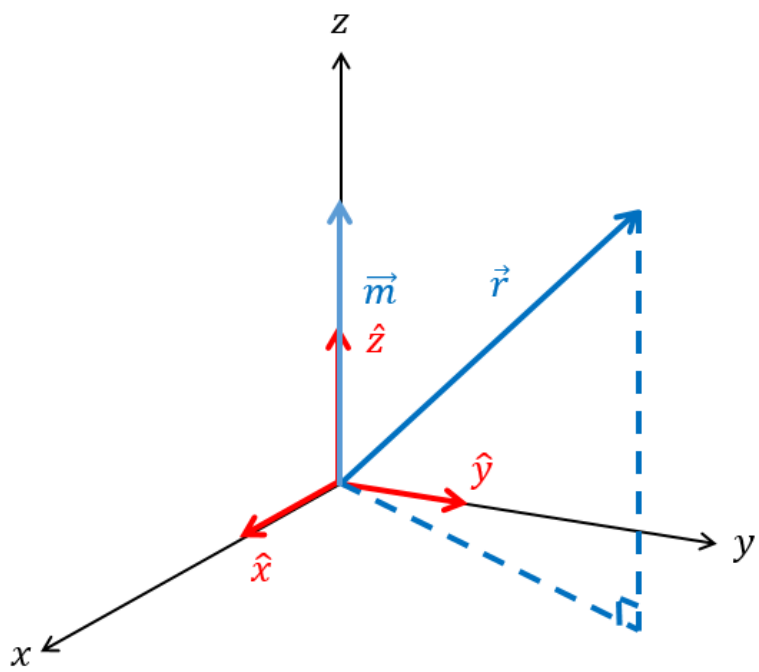

Figure a. Visualising the arbitrary vector $\vec{r}$ with respect to the vector $\vec{m}$

So $\vec{m}=m \times \hat{z}$

As a result,

$$
\begin{aligned}
& \vec{m} \cdot \vec{r} \\
= & m \times \hat{z} \cdot \vec{r} \\
= & m z
\end{aligned}
$$

We want to find $\mathrm{x}, \mathrm{y}$ and $\mathrm{z}$ components of magnetic field

$$
B_{x}=\vec{B} \cdot \hat{x}=\frac{\mu_{0}}{4 \pi} \times\left(\frac{3 \times(m z) \times \vec{r} \cdot \hat{x}}{r^{5}}-\frac{\vec{m} \cdot \hat{x}}{r^{3}}\right)
$$

As $\vec{m}$ is in $z$ direction it is perpendicular to $\hat{x}$

$$
\begin{gathered}
\therefore \vec{m} \cdot \hat{x}=0 \\
\therefore B_{x}=\frac{\mu_{0} m}{4 \pi} \times \frac{3 \times x \times z}{r^{5}}
\end{gathered}
$$

Similarly,

$$
B_{y}=\vec{B} \cdot \hat{y}=\frac{\mu_{0}}{4 \pi} \times\left(\frac{3 \times(m z) \times \vec{r} \cdot \hat{y}}{r^{5}}-\frac{\vec{m} \cdot \hat{y}}{r^{3}}\right)
$$

As $\vec{m}$ is in $z$ direction it is perpendicular to $\hat{y}$

$$
\begin{gathered}
\therefore \vec{m} \cdot \hat{y}=0 \\
\therefore B_{y}=\frac{\mu_{0} m}{4 \pi} \times \frac{3 \times y \times z}{r^{5}}
\end{gathered}
$$

On the other hand,

$$
\begin{gathered}
B_{z}=\vec{B} \cdot \hat{z}=\frac{\mu_{0}}{4 \pi} \times\left(\frac{3 \times(m z) \times \vec{r} \cdot \hat{z}}{r^{5}}-\frac{\vec{m} \cdot \hat{z}}{r^{3}}\right) \\
B_{z}=\frac{\mu_{0}}{4 \pi} \times\left(\frac{3 \times m z^{2}}{r^{5}}-\frac{m}{r^{3}}\right)
\end{gathered}
$$




$$
=\frac{\mu_{0} m}{4 \pi} \times\left(\frac{3 \times z^{2}}{r^{5}}-\frac{1}{r^{3}}\right)
$$

Now these formulas will be more helpful if we convert them into cylindrical coordinates, figure b):

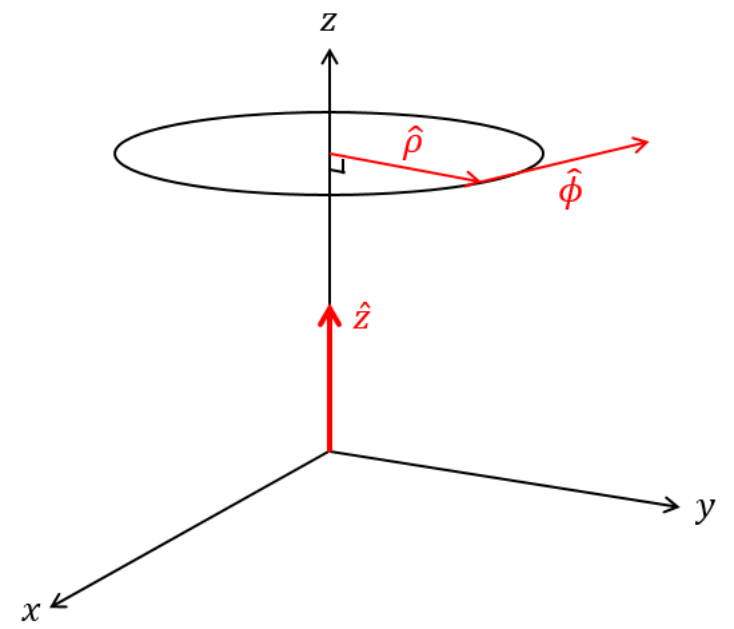

Figure b. Visualising the cylindrical coordinates that are to be used

$$
\begin{gathered}
r=\sqrt{x^{2}+y^{2}+z^{2}} \\
r=\sqrt{\rho^{2}+z^{2}}, \rho=\sqrt{x^{2}+y^{2}} \\
\vec{B}=B_{\rho} \times \hat{\rho}+B_{z} \times \hat{z}+B_{\phi} \times \hat{\phi}
\end{gathered}
$$

Due to the symmetry of the cylinder, $B_{\phi} \times \hat{\phi}=0$

$$
\begin{gathered}
\therefore \vec{B}=B_{\rho} \times \hat{\rho}+B_{z} \times \hat{z} \\
B_{\rho}=\sqrt{\left(B_{x}\right)^{2}+\left(B_{y}\right)^{2}} \\
B_{\rho}=\sqrt{\left(\frac{\mu_{0} m}{4 \pi} \times \frac{3 \times x \times z}{r^{5}}\right)^{2}+\left(\frac{\mu_{0} m}{4 \pi} \times \frac{3 \times y \times z}{r^{5}}\right)^{2}} \\
B_{\rho}=\sqrt{\left(\frac{3 \mu_{0} m z}{4 \pi r^{5}}\right)^{2} \times\left(x^{2}+y^{2}\right)} \\
B_{\rho}=\frac{\mu_{m 0} \times \frac{3 z}{4 \pi} \times \sqrt{\left(x^{2}+y^{2}\right)}}{B_{\rho}=\frac{\mu_{m 0}}{4 \pi} \times \frac{3 z \rho}{\left(\rho^{2}+z^{2}\right)^{\frac{5}{2}}}} \\
B_{z}=\frac{\mu_{0} m}{4 \pi} \times\left(\frac{3 \times z^{2}}{r^{5}}-\frac{1}{r^{3}}\right) \\
B_{z}=\frac{\mu_{0} m}{4 \pi} \times\left(\frac{3 \times z^{2}}{\left(\rho^{2}+z^{2}\right)^{\frac{5}{2}}}-\frac{1}{\left(\rho^{2}+z^{2}\right)^{\frac{3}{2}}}\right)
\end{gathered}
$$


Now with $B_{\rho}$ and $B_{z}$ we can find the emf using Faraday's law of induction:

Figure c. Visualising the significance of $d \vec{A}$

$$
\begin{aligned}
\varepsilon & =\frac{-d \phi_{B}}{d t} \\
\Phi_{B} & =\int \vec{B} \cdot d \vec{A}
\end{aligned}
$$
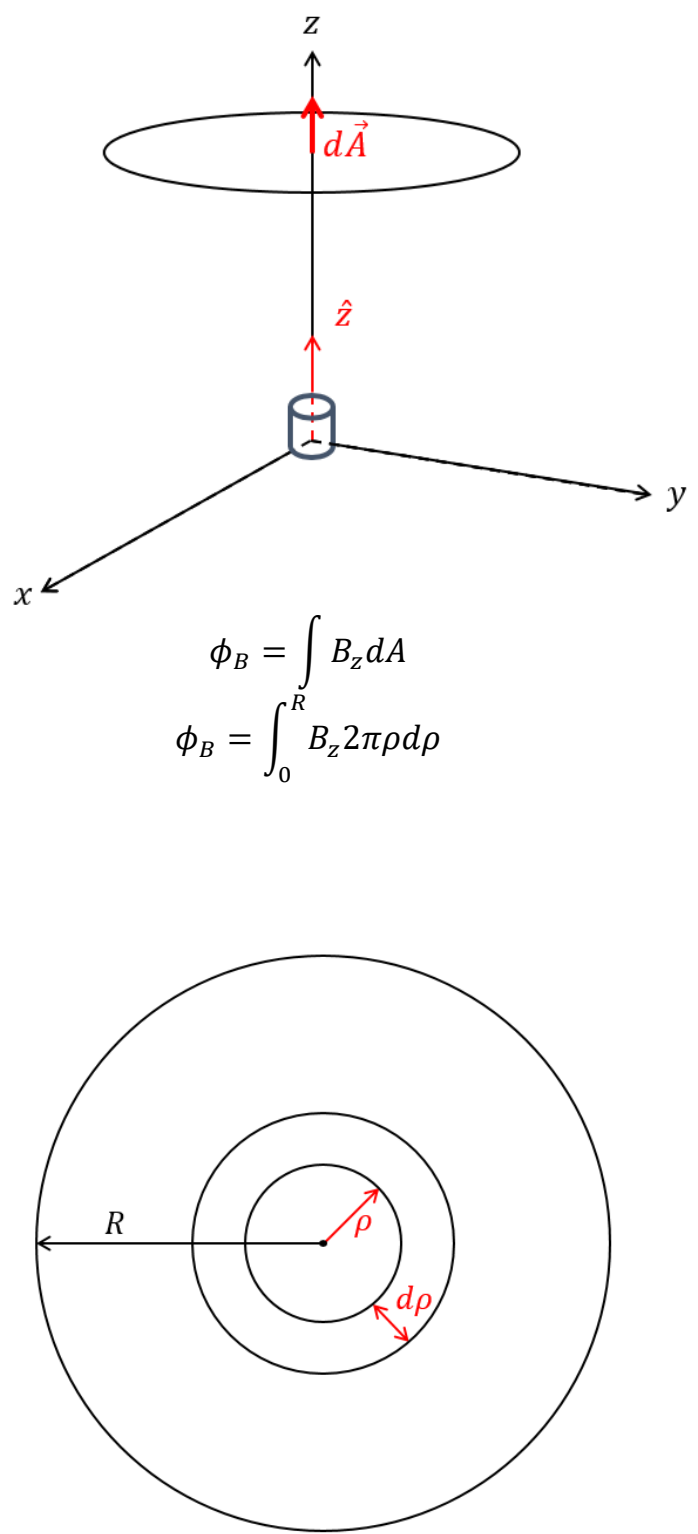

Figure d. Visualising the meaning of $\rho$ and $d \rho$

$$
\begin{gathered}
\phi_{B}=\int_{0}^{R} \frac{\mu_{0} m}{4 \pi} \times\left(\frac{3 z^{2}}{\left(\rho^{2}+z^{2}\right)^{\frac{5}{2}}}-\frac{1}{\left(\rho^{2}+z^{2}\right)^{\frac{3}{2}}}\right) \times 2 \pi \rho d \rho \\
\phi_{B}=\frac{\mu_{0} m}{4 \pi} \times \pi \times \int_{0}^{R}\left(\frac{3 z^{2}}{\left(\rho^{2}+z^{2}\right)^{\frac{5}{2}}}-\frac{1}{\left(\rho^{2}+z^{2}\right)^{\frac{3}{2}}}\right) \times 2 \rho d \rho
\end{gathered}
$$




$$
\phi_{B}=\frac{\mu_{0} m}{4} \times \int_{0}^{R}\left(\frac{3 z^{2}}{\left(\rho^{2}+z^{2}\right)^{\frac{5}{2}}}-\frac{1}{\left(\rho^{2}+z^{2}\right)^{\frac{3}{2}}}\right) \times 2 \rho d \rho
$$

To simplify this integral, the substitution $u=\rho^{2}+z^{2}$ will be used.

Derivating $u$ with respect to $\rho: d u=2 \rho d \rho$

Solving the limits, $\rho=0 \rightarrow u=z^{2}$ and $\rho=R \rightarrow u=R^{2}+z^{2}$

Now,

$$
\begin{gathered}
\phi_{B}=\frac{\mu_{0} m}{4} \times \int_{z^{2}}^{R^{2}+z^{2}}\left(\frac{3 z^{2}}{(u)^{\frac{5}{2}}}-\frac{1}{(u)^{\frac{3}{2}}}\right) d u \\
\phi_{B}=\frac{\mu_{0} m}{4} \times \int_{z^{2}}^{R^{2}+z^{2}}\left(3 z^{2} \times u^{-\frac{5}{2}}-u^{-\frac{3}{2}}\right) d u \\
\phi_{B}=\frac{\mu_{0} m}{4} \times\left[3 z^{2} \times\left(-\frac{2}{3}\right) \times u^{-\frac{3}{2}}-(-2) u^{-\frac{1}{2}}\right]_{z^{2}}^{R^{2}+z^{2}} \\
\left.\phi_{B}=\frac{\mu_{0} m}{4} \times\left[-2 z^{2} u^{-\frac{3}{2}}+2 u^{-\frac{1}{2}}\right]_{z^{2}}^{R^{2}+z^{2}}\right]_{z^{2}} \\
\phi_{B}=\frac{\mu_{0} m}{2} \times\left[-z^{2} u^{-\frac{3}{2}}+u^{-\frac{1}{2}}\right]^{R^{2}+z^{2}} \\
\left.\phi_{B}=\frac{\mu_{0} m}{2} \times\left(\frac{-z^{2}}{\left(R^{2}+z^{2}\right)^{\frac{3}{2}}}+\frac{1}{\left(R^{2}+z^{2}\right)^{\frac{1}{2}}}\right)-\left(\frac{-z^{2}}{\left(z^{2}\right)^{\frac{3}{2}}}+\frac{1}{\left(z^{2}\right)^{\frac{1}{2}}}\right)\right) \\
\left.\phi_{B}=\frac{\mu_{0} m}{2} \times\left(\left(\frac{-z^{2}+R^{2}+z^{2}}{\left(R^{2}+z^{2}\right)^{\frac{3}{2}}}\right)\right)-\left(\frac{-1}{z}+\frac{1}{z}\right)\right) \\
\left.\phi_{B}=\frac{\mu_{0} m}{2} \times \frac{\left.R^{2}+z^{2}\right)^{\frac{3}{2}}}{\left(R^{2}+z^{2}\right)^{\frac{3}{2}}}\right)
\end{gathered}
$$

Now using Faraday's daw of induction

$$
\varepsilon=\frac{-d \phi_{B}}{d t}
$$

Using the chain rule, this can be changed to $\varepsilon=\frac{-d \phi_{B}}{d z} \times \frac{d z}{d t}$ where $\frac{d z}{d t}$ is actually just the velocity.

So we get,

$$
\varepsilon=\frac{-d \phi_{B}}{d z} \times v
$$

Now we have to differentiate the magnetic flux with respect to $\mathrm{z}$.

$$
\begin{gathered}
\phi_{B}=\frac{\mu_{0} m R^{2}}{2} \times\left(R^{2}+z^{2}\right)^{\frac{-3}{2}} \\
\frac{-d \phi_{B}}{d z}=\frac{-\mu_{0} m R^{2}}{2} \times\left(-\frac{3}{2}\left(R^{2}+z^{2}\right)^{\frac{-5}{2}} \times(2 z)\right) \\
\frac{-d \phi_{B}}{d z}=\frac{3 \mu_{0} m R^{2} z}{2} \times\left(\left(R^{2}+z^{2}\right)^{\frac{-5}{2}}\right) \\
\frac{-d \phi_{B}}{d z}=\frac{3 \mu_{0} m}{2} \times \frac{R^{2} z}{\left(R^{2}+z^{2}\right)^{\frac{5}{2}}} \\
\varepsilon=\frac{3 \mu_{0} m}{2} \times \frac{R^{2} z}{\left(R^{2}+z^{2}\right)^{\frac{5}{2}}} \times v
\end{gathered}
$$

Now that we have expressions for the magnetic field and the emf we can calculate the force. 
For simplicity we are going to fix the magnetic on the origin and point it in the positive $\mathrm{z}$ direction. The ring is moving with some velocity $\mathrm{v}$ in the $\mathrm{z}$ direction. From newtons $3^{\text {rd }}$ law we know that the force exerted on the magnet due to the

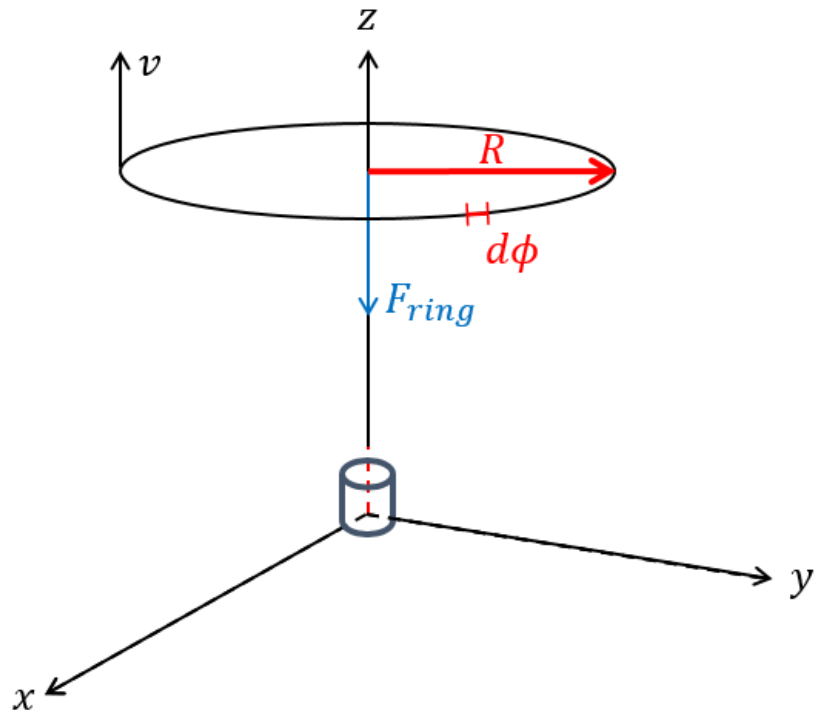

ring is the opposite of the force exerted on the ring due to the magnet. Additionally, the cylindrical symmetry of our problem means that the net force is in the $\mathrm{z}$ direction, figure $\mathrm{e}$.

Figure e. Demonstrating the direction of the net force

$$
\begin{gathered}
F_{z}^{\text {mag }}=-\int d F_{x}^{r i n g}=-\int I(d \vec{l} \times \vec{B})_{z}=\int I B_{\rho} d l \\
\int_{0}^{2 \pi} I B_{\rho} R d \phi=2 \pi R I B_{\rho}
\end{gathered}
$$

From Ohm's Law, figure f:

$$
\text { Current }(I)=\frac{\operatorname{Voltage}(V)}{\text { Resistance }(R)}
$$

Figure f. Ohm's law

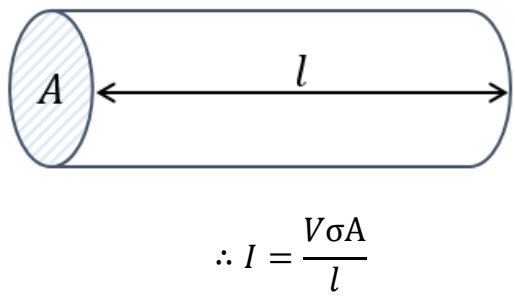

We want to find the total force acting on the magnet due to the pipe, we will do this by dividing the pipe into infinitesimally thin rings that each exert their own infinitesimally small element of force $d F$. As each ring is infinitesimally thin they have cross sectional area of $d A$. If we substitute the expression for I in, we get:

$$
\begin{gathered}
d F=2 \pi R B_{\rho} d I \\
=2 \pi R B_{\rho}\left(\frac{\varepsilon \sigma d A}{2 \pi R}\right) \\
=B_{\rho} \varepsilon \sigma d A \\
B_{\rho}=\frac{3 \mu_{m 0}}{4 \pi} \times \frac{R z}{\left(R^{2}+z^{2}\right)^{\frac{5}{2}}} \rightarrow d F=\frac{9 \mu_{0}{ }^{2} m^{2}}{8 \pi} \times v \sigma \times \frac{R^{3} z^{2}}{\left(R^{2}+z^{2}\right)^{5}} d z d r
\end{gathered}
$$


To find the total force we need to integrate $d F$ for the dimensions of the pipe, figure $g$.

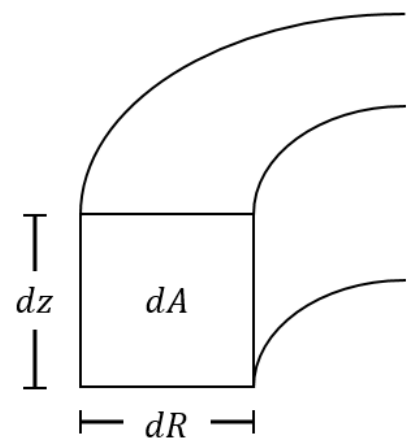

Figure g. Integrating for the dimensions of the pipe

For $R$ we are going to integrate from $a$, the inner radius of the pipe, to $b$, the outer radius of the pipe.

For simplicity we are going to assume the pipe is infinitely long and integrate $\mathrm{z}$ from $-\infty$ to $\infty$.

$$
\begin{aligned}
F & =\int d F=\frac{9 \mu_{0}{ }^{2} m^{2}}{8 \pi} v \sigma \times \int_{a}^{b} \int_{-\infty}^{\infty} \frac{R^{3} z^{2}}{\left(R^{2}+z^{2}\right)^{5}} d z d R \\
& =\int d F=\frac{9 \mu_{0}{ }^{2} m^{2}}{8 \pi} v \sigma \times \int_{a}^{b} R^{3} \times \int_{-\infty}^{\infty} \frac{z^{2}}{\left(R^{2}+z^{2}\right)^{5}} d z d R
\end{aligned}
$$

First, we will begin by solving $\alpha$.

$\alpha$

To do this, the following substitutions will be made:

$$
\begin{aligned}
z=R \tan \theta & \rightarrow d z=R \sec ^{2} \theta d \theta \\
R^{2}+z^{2} & =R^{2}+R^{2} \tan ^{2} \theta \\
& =R^{2} \times\left(1+\tan ^{2} \theta\right) \\
& =R^{2} \sec ^{2} \theta \\
R \tan \theta \rightarrow \infty \text { when } \theta \rightarrow \frac{\pi}{2} & \text { and } R \tan \theta \rightarrow \underbrace{\text { when } \theta \rightarrow-\frac{\pi}{2}}_{2}
\end{aligned}
$$

Therefore,

$$
\begin{gathered}
\alpha=\int_{-\frac{\pi}{2}}^{\frac{\pi}{2}} \frac{R^{2} \tan ^{2} \theta R \sec ^{2} \theta}{R^{10} \sec ^{10} \theta} d \theta \\
=\frac{1}{R^{7}} \times \int_{-\frac{\pi}{2}}^{\frac{\pi}{2}} \frac{\tan ^{2} \theta}{\sec ^{8} \theta} d \theta \\
=\frac{1}{R^{7}} \times \int_{-\frac{\pi}{2}}^{\frac{\pi}{2}} \sin ^{2} \theta \cos ^{6} \theta d \theta \\
=\frac{1}{R^{7}} \times \int_{-\frac{\pi}{2}}^{\frac{\pi}{2}}\left(1-\cos ^{2} \theta\right) \cos ^{6} \theta d \theta \\
=\frac{1}{R^{7}} \times \int_{-\frac{\pi}{2}}^{\frac{\pi}{2}} \cos ^{6} \theta-\cos ^{8} \theta d \theta \\
\frac{1}{R^{7}} \times\left(\int_{-\frac{\pi}{2}}^{\frac{\pi}{2}} \cos ^{6} \theta d \theta-\int_{-\frac{\pi}{2}}^{\frac{\pi}{2}} \cos ^{8} \theta d \theta\right)
\end{gathered}
$$

To solve this integral, we will define the function $f(2 n)=\int_{-\frac{\pi}{2}}^{\frac{\pi}{2}} \cos ^{2 n} \theta d \theta$.

Thus,

$$
\alpha=\frac{1}{R^{7}} \times(f(6)-f(8))
$$


Now, using integration by parts, a recurrence equation for $f(2 n)$ will be found. This equation will then be used to find a general expression for $f(2 n)$, allowing us to solve for any $\mathrm{n}$.

$$
\begin{gathered}
f(2 n)=\int_{-\frac{\pi}{2}}^{\frac{\pi}{2}} \cos ^{2 n} \theta d \theta=\int_{-\frac{\pi}{2}}^{\frac{\pi}{2}} \cos ^{2 n-1} \theta \cos \theta d \theta \\
=\left[\cos ^{2 n-1} \theta \sin \theta\right]_{-\frac{\pi}{2}}^{\frac{\pi}{2}}+\int_{-\frac{\pi}{2}}^{\frac{\pi}{2}} \sin \theta(2 n-\mathfrak{u}) \cos ^{2 n} d \tau^{2} \theta \sin \theta d \theta
\end{gathered}
$$

Now the first term in this equation is equivalent to zero:

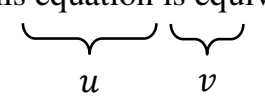

$$
\begin{aligned}
& {[\underbrace{\left.\cos _{v}^{2 n-1} \theta \sin \theta\right]_{-\frac{\pi}{2}}^{\frac{\pi}{2}} d u} d} \\
= & {\left[\frac{1}{2} \sin 2 \theta \cos ^{2 n-2} \theta\right]_{-\frac{\pi}{2}}^{\frac{\pi}{2}} }
\end{aligned}
$$$$
=\frac{1}{2}\left(\left(\sin (\pi) \cos ^{2 n-2}\left(\frac{\pi}{2}\right)\right)-\left(\sin (-\pi) \cos ^{2 n-2}\left(-\frac{\pi}{2}\right)\right)\right)
$$

$$
\begin{gathered}
=\frac{1}{2}((0)-(0)) \\
=0
\end{gathered}
$$

So we are left with,

$$
\begin{gathered}
f(2 n)=\int_{-\frac{\pi}{2}}^{\frac{\pi}{2}} \cos ^{2 n} \theta d \theta=\int_{-\frac{\pi}{2}}^{\frac{\pi}{2}} \sin \theta(2 n-1) \cos ^{2 n-2} \theta \sin \theta d \theta \\
=(2 n-1) \int_{-\frac{\pi}{2}}^{\frac{\pi}{2}} \cos ^{2 n-2} \theta \sin ^{2} \theta d \theta \\
=(2 n-1) \int_{-\frac{\pi}{2}}^{\frac{\pi}{2}} \cos ^{2 n-2} \theta\left(1-\cos ^{2} \theta\right) d \theta \\
=(2 n-1) \int_{-\frac{\pi}{2}}^{\frac{\pi}{2}} \cos ^{2 n-2} \theta-\cos ^{2 n} \theta d \theta \\
f(2 n)=(2 n-1)(f(2 n-2)-f(2 n))
\end{gathered}
$$

Solving for $f(2 n)$ :

Using this recurring formula:

$$
\begin{gathered}
f(2 n)=2 n \times f(2 n-2)-2 n \times f(2 n)-f(2 n-2)+f(2 n) \\
f(2 n)+2 n \times f(2 n)-f(2 n)=2 n \times f(2 n-2)-f(2 n-2) \\
f(2 n) \times(2 n)=f(2 n-2) \times(2 n-1) \\
f(2 n)=\frac{2 n-1}{2 n} \times f(2 n-2)
\end{gathered}
$$

$$
\begin{gathered}
f(2 n)=\frac{2 n-1}{2 n} \times \frac{2 n-3}{2 n-2} \times \frac{2 n-5}{2 n-4} \times \frac{2 n-7}{2 n-6} \times \cdots \times f(0) \\
f(0)=\int_{-\frac{\pi}{2}}^{\frac{\pi}{2}} d \theta=[x]_{-\frac{\pi}{2}}^{\frac{\pi}{2}}=\frac{\pi}{2}-\left(-\frac{\pi}{2}\right)=\pi \\
f(2 n)=\frac{(2 n) ! \div\left(2^{n} \times n !\right)}{2^{n} \times n !} \times \pi
\end{gathered}
$$

\section{Equation A2:}

$$
f(2 n)=\frac{(2 n) !}{\left(2^{n} \times n !\right)^{2}} \times \pi
$$

Using equation A2,

$$
f(6)-f(8)=\left[\frac{6 !}{\left(2^{3} \times 3 !\right)^{2}}-\frac{8 !}{\left(2^{4} \times 4 !\right)^{2}}\right] \pi
$$


Therefore,

$$
=\frac{5 \pi}{128}
$$

Therefore,

$$
\alpha=\frac{1}{R^{7}} \times \frac{5 \pi}{128}
$$

Finally,

$$
\begin{gathered}
F=\int d F=\frac{9 \mu_{0}{ }^{2} m^{2}}{8 \pi} v \sigma \times \int_{a}^{b} R^{3} \times \frac{1}{R^{7}} \times \frac{5 \pi}{128} d R \\
=\frac{9 \mu_{0}^{2} m^{2}}{8 \pi} v \sigma \times \frac{5 \pi}{128} \times \int_{a}^{b} \frac{1}{R^{4}} d R \\
=\frac{9 \mu_{0}^{2} m^{2}}{8 \pi} v \sigma \times \frac{5 \pi}{128} \times\left[\frac{-1}{3 R^{3}}\right]_{a}^{b} \\
=\frac{9 \mu_{0}^{2} m^{2}}{8 \pi} v \sigma \times \frac{5 \pi}{128} \times \frac{1}{3} \times\left(\frac{1}{a^{3}}-\frac{1}{b^{3}}\right)
\end{gathered}
$$

\section{b) Extended magnetic moment calculation}

$$
F=\frac{15}{1024} \times \mu_{0}{ }^{2} m^{2} \sigma \times\left(\frac{1}{a^{3}}-\frac{1}{b^{3}}\right) v
$$

The magnetic field $(B)$ of a magnet at a distance of $x$ meters along the axis of the magnet are related by Equation B1.

\section{Equation B1:}

$$
B=\left(\frac{\mu_{0} m}{2 \pi}\right)\left(\frac{x}{\left(x^{2}-\left(\frac{d}{2}\right)^{2}\right)^{2}}\right)
$$

where $m$ is the magnetic moment of the magnet in $\mathrm{Am}^{2}, d$ is the length of the magnet in meters (along its axis) and $\mu_{0}$ is the magnetic permeability of free space (The magnetic permeability of the smartphone and its case was assumed to be same as of free-space).

In accordance with the procedure of the article [18], my smartphone, a Samsung A70, along with the program "Physics Toolbox Magnetometer" was used to measure the magnetic field at 12 different distances. First, the position of magnetometer in phone was determined, and then smartphone was aligned with earth's magnetic field such that x-component of magnetometer is zero (magnet should be far away). Then magnet was moved along a line passing through $\mathrm{x}-$

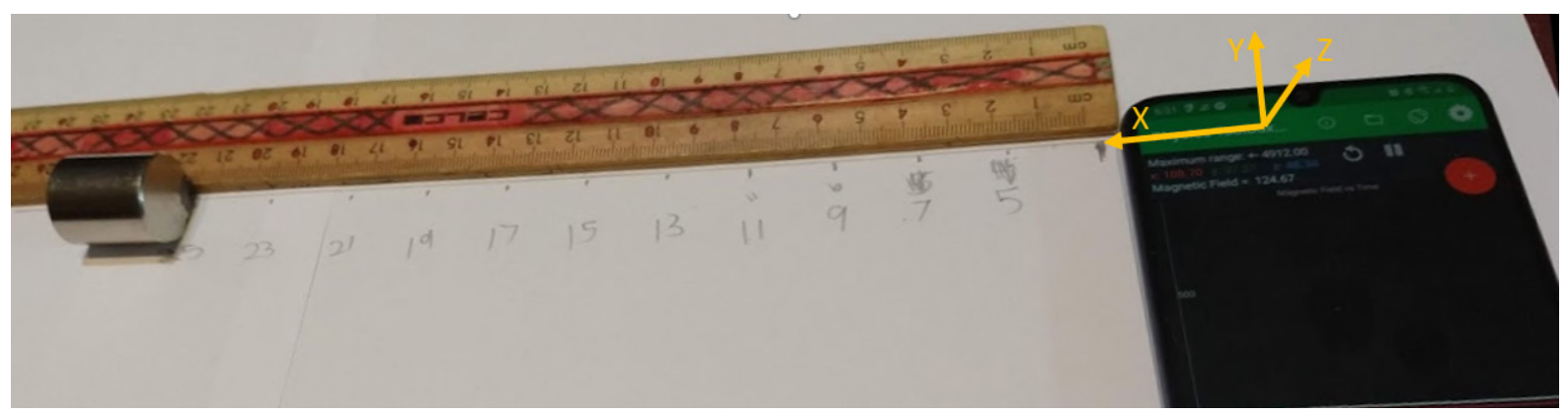

axis, figure h, and the x-component of magnetic field was recorded at 12 different distances. Figure h. The experimental setup used to measure the strength of the magnet at 12 different distances Three measurements were taken at each position and these values were then averaged, Table a. 


\begin{tabular}{|c|c|c|c|c|c|}
\hline \multirow{2}{*}{$\begin{array}{l}\text { Distance } \\
\text { (m) }\end{array}$} & \multicolumn{5}{|c|}{ Magnetic Field $(\mu \mathrm{T})$} \\
\hline & Trial 1 & Trial 2 & Trial 3 & Average & $\mathrm{SD}$ \\
\hline 0.078 & 3270 & 3170 & 3240 & 3227 & 51.316 \\
\hline 0.088 & 2320 & 2285 & 2305 & 2303 & 17.559 \\
\hline 0.098 & 1700 & 1710 & 1695 & 1702 & 7.638 \\
\hline 0.108 & 1325 & 1315 & 1295 & 1312 & 15.275 \\
\hline 0.118 & 1020 & 1010 & 1025 & 1018 & 7.638 \\
\hline 0.138 & 645 & 645 & 665 & 652 & 11.547 \\
\hline 0.158 & 455 & 425 & 450 & 443 & 16.073 \\
\hline 0.178 & 310 & 305 & 330 & 315 & 13.229 \\
\hline 0.198 & 235 & 220 & 240 & 232 & 10.408 \\
\hline 0.218 & 185 & 160 & 185 & 177 & 14.434 \\
\hline 0.238 & 140 & 125 & 150 & 138 & 12.583 \\
\hline 0.258 & 105 & 110 & 120 & 112 & 7.638 \\
\hline
\end{tabular}

Table a. Raw and processed data used to determine the magnetic moment of the magnet

The average values of the magnetic field were then plotted against the distance between magnet and magnetometer (dots) and then the non-linear least square regression function package, nls(), in $R$

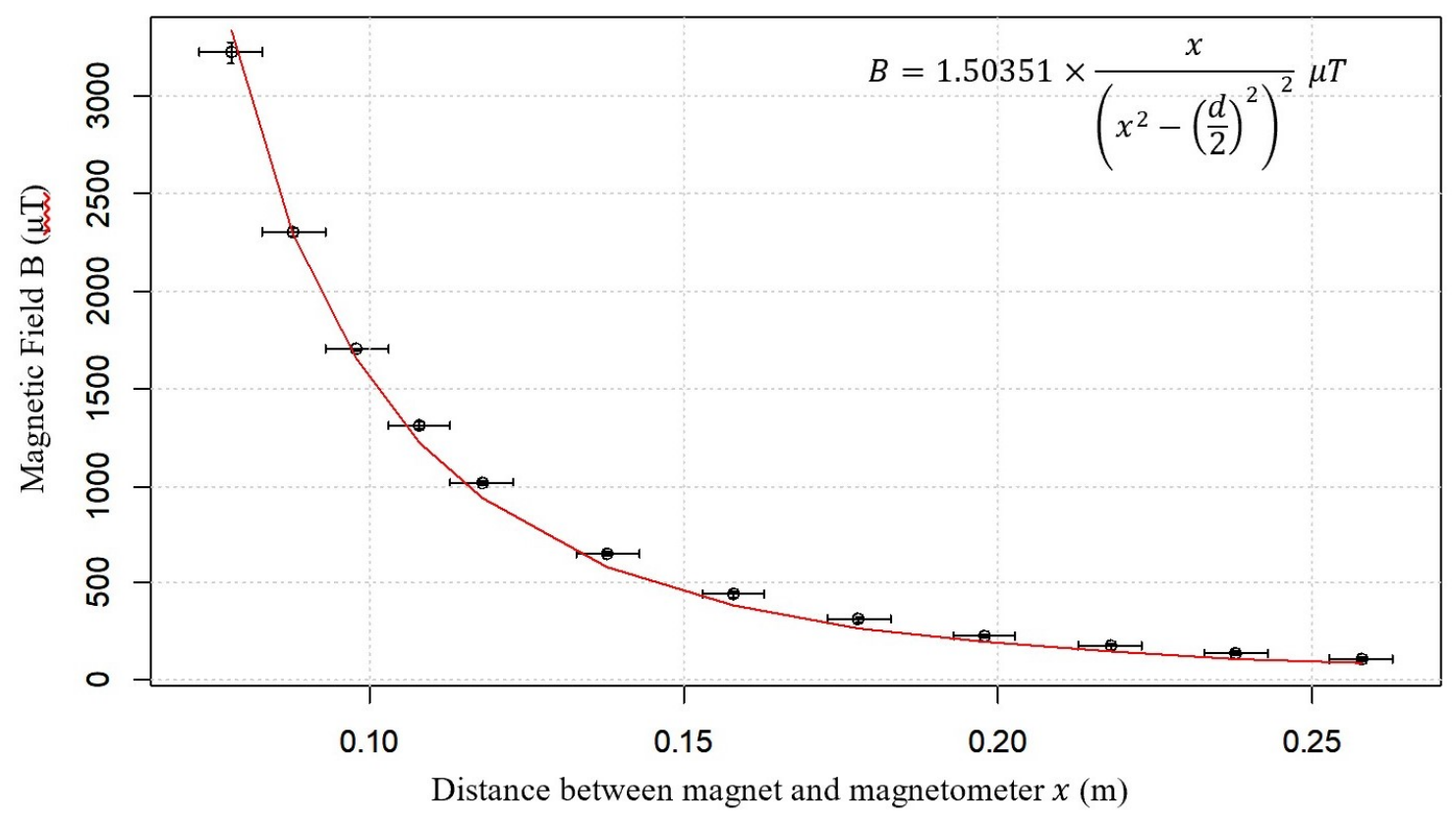

(https://rpubs.com/RobinLovelace/nls-function) on RStudio was used to fit the experimental data (red line), Figure i. Figure i. Magnetic field strength vs. Distance between magnet and magnetometer with best-fit curve fitted using the non-linear least square regression function

When fitting the data, the curve was fit according to equation B1 and $\left(\frac{\mu_{0} m}{2 \pi}\right)$ was substituted for the constant $Z$, see Equation B2. 


\section{Equation B2:}

$$
B=(Z)\left(\frac{x}{\left(x^{2}-\left(\frac{d}{2}\right)^{2}\right)^{2}}\right)
$$

The curve produced was constrained so that the only variable that was able to change was $Z$. After running the function, it produced a value for $Z$ and this value and its standard error were used to calculate the magnetic moment of the magnet and the standard error in it:

$$
\begin{aligned}
& \frac{\mu_{0} m}{2 \pi}=Z=1.50351 \pm 0.01992(S . E .) \\
& \therefore m=\frac{Z \times 2 \pi}{\mu_{0}} \pm\left(\frac{0.01992}{Z} \times 100 \%\right)=\frac{1.50351 \times 2 \pi}{4 \pi \times 10^{-7}} \pm\left(\frac{0.01992}{1.50351} \times 100 \%\right) \\
& \therefore m=7517550 \pm 1.33 \% \mu \mathrm{Am}^{2}=7.51755 \pm 0.0996 \mathrm{Am}^{2} \\
& \therefore m=7.5 \pm 0.1 \mathrm{Am}^{2}
\end{aligned}
$$

The magnetic moment could also be calculated using the equation $m=\frac{1}{\mu_{0}} \times B_{r} \times V$; where $B_{r}$ is the residual flux density of the magnet in Tesla, and $V$ is its volume in $\mathrm{m}^{3}$. $\mathrm{N} 45$ grade neodymium magnets have a residual flux density of $1.35 \mathrm{~T}$ [20]. The volume of the magnet used is $7.85 \times 10^{-6} \mathrm{~m}^{3}$.

$$
m=\frac{1}{4 \pi \times 10^{-7}} \times 1.35 \times 7.85 \times 10^{-6}=8.43 \mathrm{Am}^{2}
$$

The value of $\mathrm{m}$ based on its residual flux density is very similar to the experimentally determined value, however, the experimentally determined value could be made more accurate by using a magnetometer to measure the magnetic field rather than a smartphone.

\section{c) Determining the conductivity of the pipe at different temperatures}

The resistance of a conductor at different temperatures can be found using the resistance at a reference temperature, its temperature coefficient of resistance $(\alpha)$ and Equation $\mathrm{C} 1$.

\section{Equation C1:}

$$
R_{f}=R_{i}\left(1+\alpha\left(T_{f}-T_{i}\right)\right)
$$

where $R_{i}$ and $R_{f}$ are the resistance of the conductor at the reference temperature $\left(T_{i}\right)$ at the final temperature $\left(T_{f}\right)$ respectively.

The relationship between the resistance $(R)$ and resistivity $(\rho)$ of a material with length $l$ and cross-sectional area $A$ is described by Equation C2.

Equation

$$
R=\frac{\rho \times l}{A}
$$

C2:

Substituting Equation C2 into Equation C1, we get Equation C3.

\section{Equation C3:}

$$
\frac{\rho_{f} \times l_{f}}{A_{f}}=\frac{\rho_{i} \times l_{i}}{A_{i}}\left(1+\alpha\left(T_{f}-T_{i}\right)\right)
$$

Since the change in the length and cross-sectional area of the pipe as a result of changing its temperature is negligible, Equation $\mathrm{C} 3$ can be simplified to Equation 17.

\section{Equation 17:}

$$
\rho_{f}=\rho_{i}\left(1+\alpha\left(T_{f}-T_{i}\right)\right) ;
$$

where $\rho_{i}$ and $\rho_{f}$ are the resistivity of the conductor at the reference temperature and final temperature. 


\section{d) Full processed displacement vs. time data table}

When the temperature was very high/low, the pipe cooled down/warmed up quickly and as a result, there were less trials at the extreme temperatures.

When the standard deviation was less than the measured uncertainty of 0.04 seconds, the measured uncertainty was used instead, as shown by the cells with a blue background.

\begin{tabular}{|c|c|c|c|c|c|c|c|c|c|c|c|c|c|}
\hline \multirow{3}{*}{$\begin{array}{l}\text { Temperature } \\
\left({ }^{\circ} \mathrm{C}\right) \pm 2\end{array}$} & \multirow{3}{*}{$\begin{array}{l}\text { Position } \\
(\mathrm{m}) \\
\pm 0.006 \mathrm{~m}\end{array}$} & \multicolumn{12}{|c|}{ Time taken to reach position(s) } \\
\hline & & \multicolumn{10}{|l|}{ Trial } & \multirow[b]{2}{*}{ Mean } & \multirow{2}{*}{$\begin{array}{l}\text { Standard } \\
\text { Deviation }\end{array}$} \\
\hline & & 1 & 2 & 3 & 4 & 5 & 6 & 7 & 8 & 9 & 10 & & \\
\hline \multirow{7}{*}{-20} & 0.000 & 0.00 & 0.00 & & & & & & & & & 0.00 & 0.040 \\
\hline & 0.065 & 1.20 & 1.20 & & & & & & & & & 1.20 & 0.040 \\
\hline & 0.185 & 3.36 & 3.28 & & & & & & & & & 3.32 & 0.057 \\
\hline & 0.305 & 5.52 & 5.44 & & & & & & & & & 5.48 & 0.057 \\
\hline & 0.425 & 7.92 & 7.76 & & & & & & & & & 7.84 & 0.113 \\
\hline & 0.545 & 10.24 & 10.00 & & & & & & & & & 10.12 & 0.170 \\
\hline & 0.665 & 12.56 & 12.32 & & & & & & & & & 12.44 & 0.170 \\
\hline \multirow{7}{*}{-3} & 0.000 & 0.00 & 0.00 & & & & & & & & & 0.00 & 0.040 \\
\hline & 0.065 & 1.12 & 0.96 & & & & & & & & & 1.04 & 0.113 \\
\hline & 0.185 & 3.20 & 3.04 & & & & & & & & & 3.12 & 0.113 \\
\hline & 0.305 & 5.28 & 4.96 & & & & & & & & & 5.12 & 0.226 \\
\hline & 0.425 & 7.44 & 7.12 & & & & & & & & & 7.28 & 0.226 \\
\hline & 0.545 & 9.60 & 9.20 & & & & & & & & & 9.40 & 0.283 \\
\hline & 0.665 & 12.16 & 11.28 & & & & & & & & & 11.72 & 0.622 \\
\hline \multirow{7}{*}{3} & 0.000 & 0.00 & 0.00 & & & & & & & & & 0.00 & 0.040 \\
\hline & 0.065 & 1.04 & 0.96 & & & & & & & & & 1.00 & 0.057 \\
\hline & 0.185 & 3.12 & 2.96 & & & & & & & & & 3.04 & 0.113 \\
\hline & 0.305 & 5.12 & 4.88 & & & & & & & & & 5.00 & 0.170 \\
\hline & 0.425 & 7.20 & 6.96 & & & & & & & & & 7.08 & 0.170 \\
\hline & 0.545 & 9.28 & 8.96 & & & & & & & & & 9.12 & 0.226 \\
\hline & 0.665 & 11.28 & 11.04 & & & & & & & & & 11.16 & 0.170 \\
\hline \multirow{7}{*}{10} & 0.000 & 0.00 & 0.00 & 0.00 & 0.00 & & & & & & & 0.00 & 0.040 \\
\hline & 0.065 & 0.96 & 0.96 & 0.96 & 0.96 & & & & & & & 0.96 & 0.040 \\
\hline & 0.185 & 2.96 & 2.88 & 2.96 & 2.88 & & & & & & & 2.92 & 0.046 \\
\hline & 0.305 & 4.88 & 4.80 & 4.88 & 4.72 & & & & & & & 4.82 & 0.077 \\
\hline & 0.425 & 6.80 & 6.80 & 6.88 & 6.72 & & & & & & & 6.80 & 0.065 \\
\hline & 0.545 & 8.72 & 8.80 & 8.80 & 8.72 & & & & & & & 8.76 & 0.046 \\
\hline & 0.665 & 10.64 & 10.80 & 10.80 & 10.80 & & & & & & & 10.76 & 0.080 \\
\hline
\end{tabular}




\begin{tabular}{|c|c|c|c|c|c|c|c|c|c|c|c|c|c|}
\hline \multirow{7}{*}{15} & 0.000 & 0.00 & 0.00 & 0.00 & 0.00 & 0.00 & 0.00 & 0.00 & 0.00 & 0.00 & & 0.00 & 0.040 \\
\hline & 0.065 & 1.04 & 1.04 & 1.04 & 1.04 & 1.04 & 1.04 & 1.04 & 1.04 & 1.04 & & 1.04 & 0.040 \\
\hline & 0.185 & 2.96 & 2.96 & 2.96 & 2.96 & 2.96 & 2.96 & 2.88 & 2.96 & 2.88 & & 2.94 & 0.040 \\
\hline & 0.305 & 4.80 & 4.80 & 4.80 & 4.80 & 4.88 & 4.80 & 4.72 & 4.88 & 4.72 & & 4.80 & 0.057 \\
\hline & 0.425 & 6.80 & 6.72 & 6.72 & 6.72 & 6.80 & 6.72 & 6.64 & 6.80 & 6.56 & & 6.72 & 0.080 \\
\hline & 0.545 & 8.72 & 8.64 & 8.64 & 8.64 & 8.72 & 8.56 & 8.48 & 8.64 & 8.48 & & 8.61 & 0.089 \\
\hline & 0.665 & 10.64 & 10.56 & 10.56 & 10.56 & 10.64 & 10.48 & 10.40 & 10.56 & 10.48 & & 10.54 & 0.078 \\
\hline \multirow{7}{*}{20} & 0.000 & 0.00 & 0.00 & 0.00 & 0.00 & 0.00 & 0.00 & 0.00 & 0.00 & 0.00 & 0.00 & 0.00 & 0.040 \\
\hline & 0.065 & 0.96 & 0.96 & 0.96 & 0.96 & 0.96 & 0.96 & 0.96 & 0.96 & 0.96 & 0.96 & 0.96 & 0.040 \\
\hline & 0.185 & 2.80 & 2.80 & 2.88 & 2.88 & 2.88 & 2.88 & 2.80 & 2.80 & 2.80 & 2.88 & 2.84 & 0.042 \\
\hline & 0.305 & 4.56 & 4.64 & 4.72 & 4.64 & 4.64 & 4.64 & 4.64 & 4.64 & 4.64 & 4.72 & 4.65 & 0.045 \\
\hline & 0.425 & 6.48 & 6.56 & 6.56 & 6.48 & 6.56 & 6.56 & 6.56 & 6.56 & 6.56 & 6.56 & 6.54 & 0.040 \\
\hline & 0.545 & 8.24 & 8.40 & 8.40 & 8.40 & 8.40 & 8.40 & 8.40 & 8.40 & 8.40 & 8.40 & 8.38 & 0.051 \\
\hline & 0.665 & 10.08 & 10.24 & 10.32 & 10.24 & 10.24 & 10.24 & 10.24 & 10.24 & 10.24 & 10.32 & 10.24 & 0.065 \\
\hline \multirow{7}{*}{30} & 0.000 & 0.00 & 0.00 & 0.00 & 0.00 & & & & & & & 0.00 & 0.040 \\
\hline & 0.065 & 0.88 & 0.88 & 0.88 & 0.88 & & & & & & & 0.88 & 0.040 \\
\hline & 0.185 & 2.64 & 2.72 & 2.72 & 2.64 & & & & & & & 2.68 & 0.046 \\
\hline & 0.305 & 4.32 & 4.48 & 4.48 & 4.40 & & & & & & & 4.42 & 0.077 \\
\hline & 0.425 & 6.08 & 6.24 & 6.24 & 6.16 & & & & & & & 6.18 & 0.077 \\
\hline & 0.545 & 7.84 & 8.00 & 8.00 & 7.92 & & & & & & & 7.94 & 0.077 \\
\hline & 0.665 & 9.60 & 9.84 & 9.76 & 9.76 & & & & & & & 9.74 & 0.101 \\
\hline \multirow{7}{*}{38} & 0.000 & 0.00 & 0.00 & 0.00 & & & & & & & & 0.00 & 0.040 \\
\hline & 0.065 & 0.88 & 0.80 & 0.84 & & & & & & & & 0.84 & 0.040 \\
\hline & 0.185 & 2.56 & 2.48 & 2.60 & & & & & & & & 2.55 & 0.061 \\
\hline & 0.305 & 4.24 & 4.16 & 4.20 & & & & & & & & 4.20 & 0.040 \\
\hline & 0.425 & 6.00 & 5.84 & 5.80 & & & & & & & & 5.88 & 0.106 \\
\hline & 0.545 & 7.68 & 7.52 & 7.40 & & & & & & & & 7.53 & 0.140 \\
\hline & 0.665 & 9.36 & 9.20 & 9.08 & & & & & & & & 9.21 & 0.140 \\
\hline \multirow{7}{*}{45} & 0.000 & 0.00 & 0.00 & & & & & & & & & 0.00 & 0.040 \\
\hline & 0.065 & 0.80 & 0.80 & & & & & & & & & 0.80 & 0.040 \\
\hline & 0.185 & 2.48 & 2.48 & & & & & & & & & 2.48 & 0.040 \\
\hline & 0.305 & 4.08 & 4.08 & & & & & & & & & 4.08 & 0.040 \\
\hline & 0.425 & 5.84 & 5.68 & & & & & & & & & 5.76 & 0.113 \\
\hline & 0.545 & 7.44 & 7.28 & & & & & & & & & 7.36 & 0.113 \\
\hline & 0.665 & 9.12 & 8.96 & & & & & & & & & 9.04 & 0.113 \\
\hline
\end{tabular}




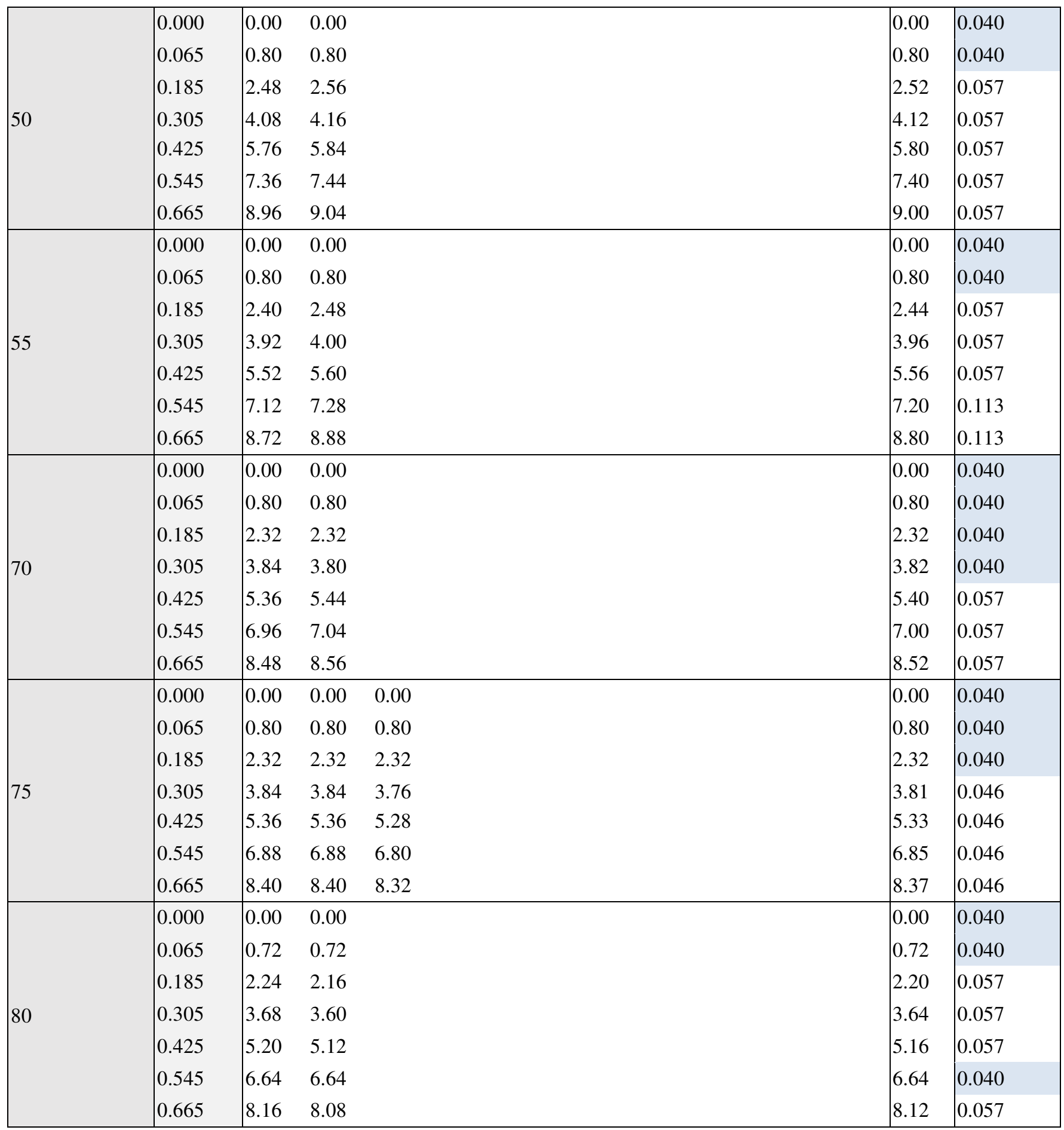

